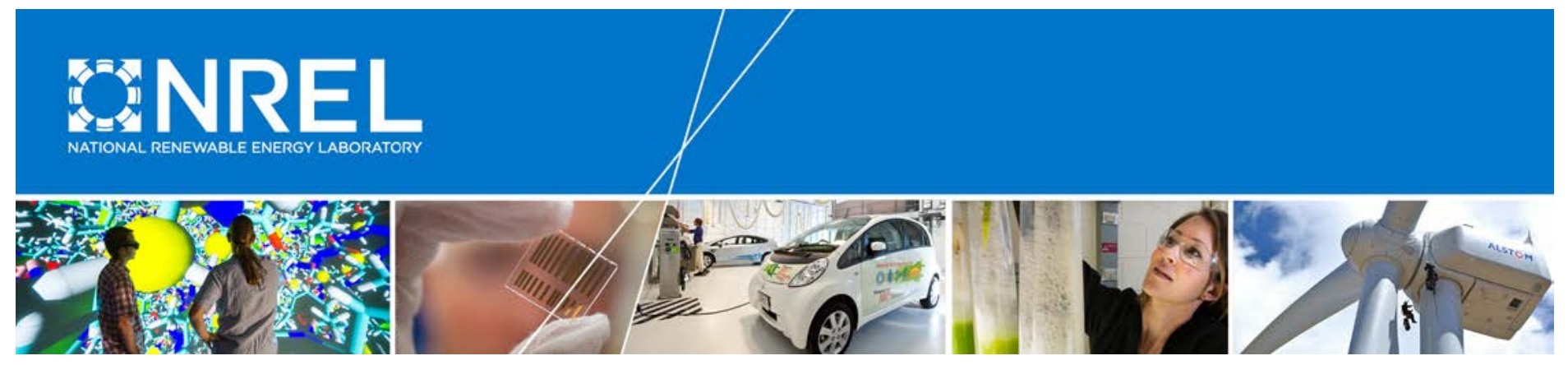

\title{
A2e High Fidelity Modeling: Strategic Planning Meetings
}

Steven W. Hammond and Michael A. Sprague National Renewable Energy Laboratory

David Womble and Matt Barone Sandia National Laboratories

This report is also published by Sandia National Laboratories. See SAND2015-9499.

NREL is a national laboratory of the U.S. Department of Energy Office of Energy Efficiency \& Renewable Energy Operated by the Alliance for Sustainable Energy, LLC

This report is available at no cost from the National Renewable Energy Laboratory (NREL) at www.nrel.gov/publications.

Technical Report

NREL/TP-2C00-64697

November 2015 


\section{A2e High Fidelity Modeling: Strategic Planning Meetings}

Steven W. Hammond and Michael A. Sprague National Renewable Energy Laboratory

David Womble and Matt Barone Sandia National Laboratories

This report is also published by Sandia National Laboratories. See SAND2015-9499.

National Renewable Energy Laboratory 15013 Denver West Parkway Golden, CO 80401

303-275-3000 • www.nrel.gov
NREL is a national laboratory of the U.S. Department of Energy Office of Energy Efficiency \& Renewable Energy Operated by the Alliance for Sustainable Energy, LLC

This report is available at no cost from the National Renewable Energy Laboratory (NREL) at www.nrel.gov/publications.

\section{Technical Report}

NREL/TP-2C00-64697

November 2015

Contract No. DE-AC36-08GO28308 


\title{
NOTICE
}

This report was prepared as an account of work sponsored by an agency of the United States government. Neither the United States government nor any agency thereof, nor any of their employees, makes any warranty, express or implied, or assumes any legal liability or responsibility for the accuracy, completeness, or usefulness of any information, apparatus, product, or process disclosed, or represents that its use would not infringe privately owned rights. Reference herein to any specific commercial product, process, or service by trade name, trademark, manufacturer, or otherwise does not necessarily constitute or imply its endorsement, recommendation, or favoring by the United States government or any agency thereof. The views and opinions of authors expressed herein do not necessarily state or reflect those of the United States government or any agency thereof.

This report is available at no cost from the National Renewable Energy Laboratory (NREL) at www.nrel.gov/publications.

Available electronically at SciTech Connect http:/www.osti.gov/scitech

Available for a processing fee to U.S. Department of Energy and its contractors, in paper, from:

\author{
U.S. Department of Energy \\ Office of Scientific and Technical Information \\ P.O. Box 62 \\ Oak Ridge, TN 37831-0062 \\ OSTI http://www.osti.gov \\ Phone: 865.576.8401 \\ Fax: 865.576.5728 \\ Email: reports@osti.gov
}

Available for sale to the public, in paper, from:

\author{
U.S. Department of Commerce \\ National Technical Information Service \\ 5301 Shawnee Road \\ Alexandria, VA 22312 \\ NTIS http://www.ntis.gov \\ Phone: 800.553 .6847 or 703.605 .6000 \\ Fax: 703.605.6900 \\ Email: orders@ntis.gov
}




\title{
ATMOSPHERE TO ELECTRONS
}

A2e High Fidelity Modeling: Strategic Planning Meetings

\author{
ModSim Environment
}

27-28 January 2015, Denver, CO

Wind Plant Physics and Modeling

24-25 February 2015, Crystal City, Washington, DC 


\section{Atmosphere to Electrons Initiative - High Fidelity Modeling ModSim Environment, and Wind Plant Physics and Modeling}

\section{Planning Meeting Report}

\section{Planning Meeting Co-Chairs}

David Womble, Sandia National Laboratories

Steve Hammond, National Renewable Energy Laboratory

\section{ModSim Environment, Program Committee}

Michael Sprague, National Renewable Energy Laboratory (Program Chair)

Ramesh Balakrishnan, Argonne National Laboratory

Kevin Barker, Pacific Northwest National Laboratory

James Stewart, Sandia National Laboratories

John Turner, Oak Ridge National Laboratory

\section{Wind Plant Physics and Modeling, Program Committee}

Matt Barone, Sandia National Laboratories (Program Chair)

Jim Brasseur, Penn State University

Rod Linn, Los Alamos National Laboratory

David Maniaci, Sandia National Laboratories

Pat Moriarty, National Renewable Energy Laboratory

Jeff Mirocha, Lawrence Livermore National Laboratory

Greg Oxley, Vestas

Dominic von Terzi, GE Global Research

Fotis Sotiropoulos, University of Minnesota

\section{DOE Office of Energy Efficiency and Renewable Energy Contacts}

Michael Robinson, DOE Wind Program

Shreyas Ananthan, DOE Wind Program 


\section{Executive Summary}

The U.S. Department of Energy (DOE) Wind Program is committed to developing and deploying a portfolio of innovative technologies for clean, domestic power generation, targeting scenarios where wind energy accounts for $35 \%$ of our nation's electricity supply by 2050 [1]. The Program's activities are leading the nation's efforts to accelerate the deployment of wind power technologies through improved performance, lower costs, and reduced market barriers. The Program works with industry, national laboratories, universities, and other federal agencies to conduct research and development activities. Greater use of the nation's abundant wind resources for electric power generation will strengthen U.S. energy security through greater diversity in its energy supply, provide cost-competitive electricity to key regions across the country, and reduce water used in thermo-electric power generation. In addition, wind energy deployment will help stimulate the revitalization of key sectors of the economy by investing in infrastructure and creating long-term, sustainable skilled jobs.

There is a well-established wind industry led by large, multinational corporations. This industry has been successful in building blades and turbines and deploying them as wind plants composed of multi-turbine arrays. At present, approximately $5 \%$ of electricity generated in the U.S. comes from wind and the cost per kilowatt hour is competitive with other traditional fuel sources. However, plant-level energy losses are estimated to be $20 \%$ and up to $30 \%$ in areas of complex terrain. It is estimated that just a $1 \%$ improvement would result in over $\$ 100$ Million dollars annually at present penetration levels. As wind generated electricity approaches $20 \%$ to $30 \%$ of U.S. electrical supply, a 2\% improvement in efficiency would save over \$1 Billion annually.

Atmosphere to electrons (A2e) is a multi-year U.S. Department of Energy (DOE) research initiative targeting significant reductions in the cost of wind energy through an improved understanding of the complex physics governing wind flow into and through whole wind farms. Better insight into the flow physics of large multi-turbine arrays will address the plant-level energy losses, is likely to reduce annual operational costs by hundreds of millions of dollars, and will improve project financing terms to more closely resemble traditional capital projects.

In support of this initiative, two planning meetings were convened. These two-day meetings brought together professionals from universities, national laboratories, and industry to discuss wind plant modeling challenges, requirements, best practices, and priorities. This report documents the combined work of the two meetings and serves as a key part of the foundation for the A2e/HFM effort for predictive modeling of whole wind plant physics.

The first planning meeting focused on requirements and priorities for developing a predictive wind plant modeling and simulation environment. Participants assessed the state of the art in open source community codes, large-scale numerical modeling, and developed requirements and specifications for a high-fidelity modeling and simulation environment. The second planning meeting focused on wind plant physics and modeling. Participants identified prioritized quantities of interest at various scales, the experimental data necessary to validate these quantities and re-addressed software needs from a wind energy modeling perspective. Full details of the two planning meetings are in the sections that follow.

These two planning meetings build on earlier wind energy and related workshops, studies and planning documents [1-8]. This summary report differs from these other reports and efforts in that the focus here is on priorities and requirements for building the high-fidelity modeling and simulation environment necessary to study the fundamental flow physics of whole wind plants. The other reports focused on the "what" and "why" and here it is more focused on the "how."

The challenge is daunting - to enable alternative and renewable energy technologies to achieve their potential in meeting future energy needs. However, high-performance computing and 
modeling can play a significant and dramatic role in accelerating the required research.

Fortunately, we are seeing continued rapid growth in computational capability and favorable reductions in the cost of large-scale simulation. Together, these two trends are enabling substantial simulation-based research and development breakthroughs in numerous fields of science, technology, and engineering.

Addressing the computational research needs identified in this report will reap tremendous benefits in terms of advancing the basic science underlying alternative and renewable energy technologies. This will lay the groundwork for, and greatly accelerate, the scientific and technological advances that will play a major role in meeting global energy needs - sustainably and cost effectively. 


\section{Table of Contents}

Executive Summary ...................................................................................................................... iii

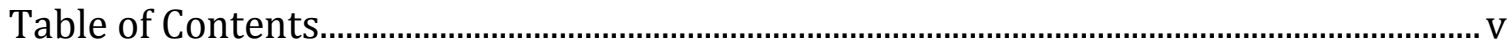

1 Introduction and Background...................................................................................

2 Modeling and Simulation Environment Planning Meeting ...........................................

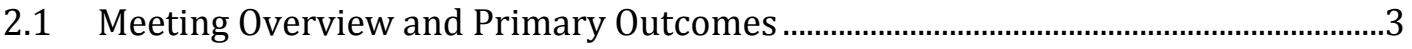

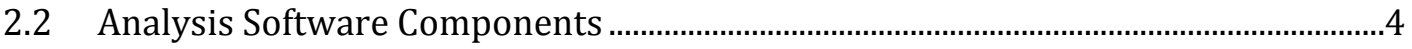

2.3 Implementation of Flow Physics and Numerical Discretization of PDEs..................7

2.4 Addressing Software Engineering, Performance, and Scalability Issues on the

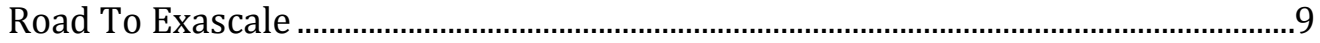

2.5 Infrastructure, Integration, and Software Development Processes .........................14

3 Wind Plant Physics and Modeling ..............................................................................2

3.1 Meeting Overview and Primary Outcomes ...................................................................21

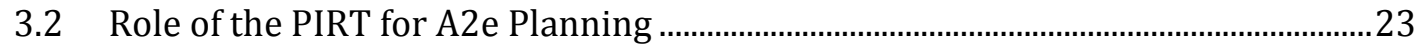

3.3 Breakout: Mesoscale and Mesoscale/Plant Scale Interface ........................................26

3.4 Breakout: Plant Scale and Plant Scale/Turbine Scale Interface .................................2

3.5 Breakout: Turbine Scale, Blade Chord Scale, and Turbine/Blade Chord Interface 32

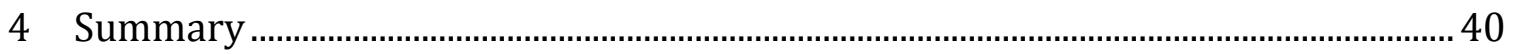

1 Appendix: Related Documents \& Reports ………………........................................ 42

2 Appendix: ModSim Environment Strategic Planning Meeting Agenda.................... 43

3 Appendix: Wind Plant Physics and Modeling Planning Meeting Agenda ............... 45

4 Appendix: January Planning Meeting Registered Attendees..................................... 48

5 Appendix: February Planning Meeting Registered Attendees....................................5 50 


\section{Introduction and Background}

Wind power is one of the fastest-growing sources of new electricity supply and the largest source of new renewable power generation added in the United States since 2000. Wind power generation in the United States has more than tripled in recent years, increasing from $1.5 \%$ of annual electricity end-use demand in 2008 to 5\% through 2014. As of 2013, there were more than 61 gigawatts $(\mathrm{GW})$ of wind generating capacity installed [1]. Wind energy is also the technology of choice in countries around the world for improving energy security, job creation, and reducing $\mathrm{CO}_{2}$ emissions, as it is clean, reliable, quick to install, and requires no water. Although wind energy holds immense promise, high-penetration wind energy deployment raises fundamental science questions concerning potential environmental and weather-related impacts, policy questions concerning U.S. energy strategy and regulations, and financial questions.

Computational-simulation technologies are widely used in the wind industry. However, they have focused on design and performance of single turbines and are limited in their ability to predict and optimize the dynamic and nonlinear processes and phenomena within and between large-scale wind farms, or between wind farms and the environment. High-penetration wind energy deployment will entail extracting elevated energy levels from the planetary boundary layer and preliminary studies indicate this will have significant but uncertain impacts on the local and regional environment. Assessing impacts and quantifying uncertainties in high penetration wind scenarios requires the ability to analyze and predict the behavior of multiscale simulation models able to address spatial regimes spanning ten orders of magnitude (from turbine blade aerodynamics through large-scale atmospheric processes). This is computationally demanding and will both require and leverage U.S. investments in exascale class computing.

The focus of the A2e High Fidelity Modeling (HFM) focus area is to understand and accurately model fundamental physics on appropriate temporal and spatial scales to predict complex, rapidly changing inflow to the wind plant, complex flow within a wind plant, and the response of individual turbines and the wind plant as a whole to the flows utilizing high-fidelity modeling. This will require High Performance Computing (HPC) modeling and simulation tools developed in a community-based, open-source simulation environment. These tools will be validated using measurements acquired in research grade experiments/tests specifically aimed at credibly assessing computational accuracy within a Verification and Validation (V\&V) paradigm. The measurements will incorporate emerging remote sensing and other technologies that represent the state of the art in quantifying flow fields around and within wind plants.

Two planning meetings were held during January and February of 2015. The goals of these meetings were the following:

1. Engage both the wind plant modeling community and the high-performance computing community to assess the current state of simulation tools, algorithms and capabilities for wind-plant scale simulations and plant performance predictions.

2. Discuss tools and methodology available for verification and validation and apply these tools (e.g., PIRTs) to identify linkages and dependencies between modeling and simulation and experimental measurements that must be filled before simulation can be used confidently in decisions.

3. Identify gaps in both modeling and simulation capabilities that will require investments over the next five to ten years.

4. Identify key actions and investments that are required to develop an open-source simulation infrastructure. 
5. Identify challenges, issues and best practices for developing a community-based approach to high-fidelity modeling and simulation.

The next two sections of this report summarize the specific outcomes from these two planning meetings. Each section includes summaries of the planning, descriptions of the structure of the meetings and detailed notes from each of the breakout sessions. 


\section{Modeling and Simulation Environment Planning Meeting}

\subsection{Meeting Overview and Primary Outcomes}

The Modeling and Simulation (ModSim) Environment Strategic Planning Meeting brought together about 70 professionals from universities, national laboratories, and industry to discuss challenges and best practices for developing robust community modeling environments. Meeting participants assessed the state of the art, and developed requirements and framework specifications for a high fidelity ModSim environment that will be the foundation for A2e HFM predictive modeling of whole wind plant physics.

The meeting included breakout groups that were inter-woven with invited plenary talks and discussion. The guiding principles for defining the ModSim environment were shared with participants at the meeting's outset. These guiding principles are that the HFM ModSim environment will:

1. Be the foundation for state-of-the-art predictive and physics-based simulations of whole wind plants,

2. Value high fidelity, performance, and ability to effectively exploit large core,

3. Leverage existing assets where appropriate and develop new resources as needed,

4. Be designed to accommodate future exascale systems,

5. Target simulations that aspire to "ground truth", and solutions will have known confidence intervals, based on rigorous verification and validation, and

6. Be an open-source community model whose contributors and users will be from national labs, universities, and industry.

Plenary speakers gave concise and compelling talks that informed the ModSim Environment discussion. Talk titles and speakers were as follows:

1. Atmosphere to Electrons, Mike Robinson, US DOE Wind Program

2. The role of high fidelity modeling in A2e, Shreyas Ananthan, US DOE Wind Program

3. Trends in HPC environments, Paul Messina, ANL

4. The treatment of turbulence and boundaries in wind farm simulations, Philippe Spalart, Boeing, and Matt Churchfield, NREL

5. Wind farm simulations using geometry-conforming models for individual wind turbines, Jay Sitaraman, U. of Wyoming and U.S Army at NASA Ames

6. Creating a flexible, extensible and maintainable Community Earth System Model, Mariana Vertenstein, NCAR

7. Multi-Lab collaborative development of ACME, Mark Taylor, SNL

8. Multi-Institutional Development of Scientific Software - Lessons Learned in CASL, John Turner, ORNL

9. Uncertainty and Reliability in Computational Predictions, Robert Moser, U. of Texas at Austin

The four breakout topics and important outcomes are summarized below, with detailed discussion following in subsequent sections.

\subsubsection{Analysis software components:}

The Analysis Software Components breakout group identified five priority research topics: (i) UQ/Sensivity Analysis, (ii) verification, (iii) coupling computation and physical data, (iv) data analysis, and (v) multi-fidelity model management. Two overlapping needs emerged:

the need to develop predictive computational models of relevant physics and their couplings, with well-characterized uncertainties, supported by physical data and the need to address the extreme 
scales of a predictive high-fidelity modeling capability. Outer-loop simulations (e.g., UQ, design optimization, data assimilation, model calibration, etc.) generally require exploration of a very high dimensional space (need for many simulation runs). The challenge is to obtain reliability approaching that of the highest-fidelity simulations at a realistically achievable cost. This requires advancements in UQ and inverse analysis algorithms, data analysis, and a multi-fidelity modeling framework. Results of this breakout are described in detail in Section 2.2.

\subsubsection{Implementation of flow physics and numerical discretization of PDEs}

This breakout group defined the underlying flow model and appropriate numerical-solution methods that leverage DOE-supported open-source tools that adequately solve the whole wind plant system on today's petascale and tomorrow's exascale computer systems. The motivation in making these decisions early on was that the choice of flow model and associated numericaldiscretization methods (e.g., spatial and temporal discretization) would greatly influence the nature, performance, and capabilities of the ModSim environment. A detailed discussion of recommendations is provided in Section 2.3.

\subsubsection{Addressing software engineering, performance, and scalability issues on the road to exascale}

This breakout group identified the software engineering and design challenges and solutions in constructing a community simulation code that will bridge disparate time and length scales, and will also scale to future exascale-sized computing systems. Topics covered included elevating performance prediction and modeling to a "first-class citizen" of the software design process, software design decisions that will impact scalability to possibly billion-way fine-grained parallelism, and potential strategies to address the challenges posed by advanced architectures. A detailed discussion of the challenges that must be addressed in order to achieve exascale computing is provided in Section 2.4.

\subsubsection{Infrastructure, integration, and software development processes}

This breakout group identified the challenges inherent in the collaborative development of a large complex software product with a geographically dispersed, multi-institutional team. These include both technical and non-technical challenges. Specific topics will include software ownership and licensing, robust build and test systems, developer workflow, software lifecycle (balancing research and production), and infrastructure resource requirements (both hardware and staffing). A detailed discussion of the challenges and processes for addressing those challenges, along with lessons learned from similar programs is provided in Section 2.5.

\subsection{Analysis Software Components}

Chair: James Stewart (SNL) Co-Chair: Paul Constantine (Colorado School of Mines)

\subsubsection{Breakout description and summary}

The Analysis Software Components breakout focused on the "outer loop" technologies including (but not necessarily limited to) uncertainty quantification, data assimilation, meshing, visualization, data mining, optimization, etc. Specifically, the group surveyed current approaches and tools with respect to our overarching goal of a community-based predictive modeling capability, and then identified research and development gaps/challenges, development priorities, possible use cases, and computational-resource requirements.

Planning for the analysis software components of the A2e high-fidelity ModSim effort is dependent on how the high-fidelity simulations are to be used in the larger A2e program. There 
are a number of critical roles such simulations might play, and these were explored in the breakout discussions to set the context for recommendations regarding analysis software components. In simulating a complex physical system such as a wind plant, the objectives will generally require that a high-dimensional space of possible inputs be explored. This may be for uncertainty quantification, design optimization, data assimilation or model validation. Such explorations require that the simulation model be run many times, putting practical limits on both the computational cost of each simulation and the overall fidelity of the simulation. By lifting the requirement to perform many simulations, it is of course possible to perform much higher-cost and higher-fidelity simulations. The challenge is to effectively use the simulation models over a spectrum of fidelities and costs to obtain the programmatically required results with reliability approaching that of the highest-fidelity simulations.

Accomplishing this will require a multi-fidelity simulation capability, in which high-fidelity simulations are used to inform and characterize errors in lower-cost models, which are used to explore the input spaces discussed above. To see how this might work, it is useful to borrow the concept of the "validation pyramid." High-fidelity models of systems with lower complexity than an entire wind plant will be of great value in calibrating and testing lower-fidelity models. In the simplest cases (e.g. a single turbine, or even a single blade) many high-fidelity simulations can be used in conjunction with experimental data to inform lower-fidelity models. Going up the pyramid (e.g. two interacting turbines) will allow other aspects of lower-fidelity models to be informed, with fewer high-fidelity simulations. At the top of the pyramid might be a full wind plant, for which perhaps a single carefully chosen high-fidelity simulation could be done to address particular plant-scale modeling issues in lower-fidelity representations. Another important interaction between low- and high-fidelity models would be when a low-fidelity model moves into a regime in which the model has not been well tested and characterized, or when it is producing unexpected results. In this case, high-fidelity simulation(s) for a smaller system abstracted to expose the issue at hand would be used to address the issue. These and similar use cases will require integrated use of high- and lower-fidelity simulations, and this is the context in which the analysis software component recommendations below are made.

The analysis software components breakout emerged with five priority research topics: (1) Uncertainty Quantification and Sensitivity Analysis, (2) Verification, (3) Coupling Computation and Physical Data, (4) Data Analysis, and (5) Multifidelity Model Management. The emergence of these five topics reflects the A2e/HFM focus on predictive modeling of whole wind plant physics, while acknowledging the role of a multifidelity modeling capability as a means of achieving this (as discussed above). While there is some overlap among these topics, each involves significant and unique challenges. Two overlapping themes among most or all of the areas include the following: First, there is the need to develop predictive computational models, with well-characterized uncertainties, of the relevant physics and their couplings, supported by physical data. Second, A2e/HFM must address the extreme scales of a predictive high-fidelity modeling capability. Such scales exist in the multiscale, multiphysics whole wind plant model (both time and spatial scales), the uncertainties that are present from all sources (e.g., input conditions, boundary loads, material properties, model selection, etc.), and the data (including simulation inputs and outputs, as well as experimental data). In addition to the extreme scale of data, A2e/HFM must also deal with highly complex data that is possibly incomplete and/or difficult to characterize statistically.

We proceed with a brief summary of each of the priority research topics.

Uncertainty quantification and sensitivity analysis.

This topic is characterized by a range of methods that may involve "black-box" sampling of the random input space, or more advanced methods that involve some degree of intrusiveness into the 
simulation code. Methods exist for addressing both aleatory and epistemic uncertainties. The more robust methods are of the sampling type, but they also tend to exhibit slow convergence. More advanced methods have been shown to greatly improve performance, but often require overly restrictive assumptions and/or are difficult to implement. Potential research directions include: (1) Extreme high random dimensions of $O\left(10^{2}-10^{6}\right)$, (2) Extreme scale of the forward simulation (which can severely limit the number of simulation runs that are possible), (3) Uncertainties due to model form or inadequacy, (4) Design under uncertainty, (5) Nonsmooth or chaotic quantities-of-interest, (6) Exploiting derivative information from the computational models.

Requirements emanating from this topic include the following: (1) Quantities-of-interest should be specified such that UQ and sensitivity analysis are well defined, (2) All potential sources of uncertainty should be identified, (3) All uncertain models and parameters should be exposed in the simulation input, (4) Adjoints and/or derivatives (of simulation outputs with respect to inputs) should be provided when possible, (5) Execution times for a single simulation run should be specified. In the near term (1-3 years), addressing this research topic will lead to the ability to develop new, UQ-enabled computational models (e.g., with well-characterized and reduced uncertainties). In the longer term ( $>3$ years), it is expected that predictions of quantities-ofinterest at HFM scales will be achievable. This implies that extrapolations beyond experimental conditions, with quantified credibility statements, will be possible. Also in the longer term, this research topic could lead to impact on design standards, load estimates (on the power grid), and annual energy production estimates.

\section{Verification}

Support for verification is a critical requirement of any software environment for modeling and simulation, and there are well-established verification techniques that can be deployed. The verification of code correctness can generally be verified through code-to-code comparisons, the method of manufactured solutions, and measurements of convergence rates. The verification that solutions are adequately converged can be accomplished through such techniques as a posteriori error estimation, analysis of uniform and adaptive grid refinement or coarsening, and for statistical quantities. One issue that does need to be addressed in solution verification is the treatment of LES regions, in which the model definition is commonly tied to the grid size. As in all computational science problems, code and solution verification are critical to establishing the credibility of the computations and the resulting predictions. To enable code and solution verification, there are several requirements for the simulation codes. These include the ability to refine grids uniformly and/or adaptively, support for numerical error estimators, and stable, accurate and convergent computational models. Further, in the context of uncertainty quantification, it is important that solution-verification error expectations be informed by expected levels of uncertainty. Ideally, numerical errors should be small compared to uncertainties, but need not be extremely small.

\section{Coupling computation and physical Data}

The overarching goal of this topic is to impact how new models are developed. If successful, the short-term impact will be the ability to develop new, data-informed models with reduced uncertainties. The algorithmic need encompasses data assimilation (e.g., Kalman filters), calibration of model parameters (e.g., least squares fitting, Bayesian calibration, etc.), and computation-informed optimal design of experiments. Research directions include model inadequacies (or discrepancies), characterization of uncertainties in both computations and experiments, insensitivities of the models with respect to the data (i.e., identifiability), and extreme high dimensions (e.g., in parameter space). Related to this is the need to define relevant validation metrics, sufficiently characterize experimental and simulation input uncertainties 
(including specification of priors), and extract quantities-of-interest from experiments. Close collaboration between experimentalists and computational scientists is expected. In the longer term, this collaboration will lead to new, predictive models with reduced uncertainties and/or well-characterized limitations.

Data analysis

In this area, the A2e/HFM program will require capacity for input/output and storage of extremescale datasets. A common database that is accessible throughout the A2e community will also be needed. A2e researchers and analysts will require the ability to manage large ensembles of runs (which in turn generate extreme volumes of data) and extract features from the datasets. In situ and postprocessing diagnostics capabilities will be critical. The research directions span these areas: Data reduction, data mining, and in situ data analytics. Short term expected impacts from the data analysis area include the ability to routinely share data across A2e, and the ability to identify modeling targets (e.g., features) that are observant in data or required in simulations (a modeling target might include, for example, an increased resolution of an underperforming turbine or an increased fidelity for a plant siting). A longer term expected impact is on future siting and/or optimization of plant designs, as well as on operational strategies.

\title{
Multifidelity model management
}

The motivation for this priority research topic was discussed previously. The challenge, also stated previously, is to effectively use the simulation models over a spectrum of fidelities and costs to obtain the programmatically required results with reliability approaching that of the highest fidelity simulations. This requires the a priori knowledge of the high-fidelity "run budget" (i.e., how many runs can we afford?), the ability to save data from high-fidelity models to inform low-fidelity models, and the identification of compatible low-fidelity model forms. The ability to manage ensembles of discrete models is also needed which, in turn, requires consistencies in model parameterization, quantities of interest, etc. Multifidelity model management includes important research topics, e.g., goal-oriented adaptive modeling, combined multifidelity UQ and optimization, grid refinement in the context of LES models, and the broad theme of developing new high-fidelity model forms that can optimally inform engineering/design scale studies. In the short term, the impact will be the creation of useful low-fidelity models that are generated from very few high-fidelity simulations (for the likely situation where small highfidelity run budgets exist). In the long term, the multifidelity modeling approach will lead to a credible set of high-fidelity simulations that are useful for validation (where validation data exists) and certification (i.e., predictions that require extrapolations beyond conditions where data exists).

\subsection{Implementation of Flow Physics and Numerical Discretization of PDEs}

\author{
Chair: Ramesh Balakrishnan (ANL) Co-Chair: Stefan Domino (SNL)
}

\subsubsection{Description and summary}

This breakout focused on the numerical discretization techniques being applied to the fluid- and structural-dynamics equations and their impacts on: a) the physics that are being resolved, and b) the scaling and parallel performance of the compute engine in the ModSim environment. There are quite a few well-known codes, and numerical techniques, such as the finite-volume, finitedifference, and continuous/discontinuous Galerkin schemes on structured (overlapping) and unstructured meshes. Further, there is a veritable wealth of literature that documents the performance of these numerical schemes, when applied to problems that are ideal, in the sense that problem sets to which they have been applied are (for the most part) those where one has a fairly large degree of control, ranging from the specification of the flow conditions to those of the 
geometry. However, when one applies the very same numerical techniques to drive the compute engine in a predictive-simulation tool, with considerable uncertainties in the flow conditions, and complexities in the geometry, it is not quite clear which of the competing methods could serve as the gold standard (and continue to evolve as one). Further, there is the software engineering aspect of designing the compute engine in ModSim for exascale platforms, where it is becoming clear that one will need to explore (and exploit) fine grain parallelization techniques, in addition to the tried and tested MPI paradigm, to scale the code on $O\left(10^{6}\right)$ cores. And, finally, we discussed the development of APIs to enable the use of ModSim by other researchers with custom built (and, perhaps, proprietary) modules.

The Implementation of Flow Physics and Numerical Discretization of PDEs panel defined an underlying flow model and appropriate numerical-solution methods that will leverage DOEsupported open-source tools that adequately solve the whole wind plant system on today's petascale and tomorrow's exascale computer systems. The path described below is not necessarily optimal, but the panel has great confidence that it will be successful because it is built on production-hardened methods and DOE future-supported libraries for today's and tomorrow's HPC.

\subsubsection{Mathematical models}

For the wind plant scale, the flow will be modeled as time-dependent (unsteady) incompressible Boussinesq, and turbulence will be modeled as smoothly blended detached eddy simulation (DES). In DES, near-turbine-surface flow will be solved in Reynolds Averaged Navier Stokes (RANS) whereas off-turbine flow will be solved with dynamic Large-Eddy Simulation (LES) with wall models near the ground/water interface. At the highest fidelity, simulations will resolve the blade/nacelle/tower in the fluid domain. Reduced-fidelity simulations, where the blade is represented as a state-of-the-art "actuator line," will be pursued concurrently. For the meso scale, existing and future numerical weather prediction tools and atmospheric-boundary-layer simulation tools will be employed (with their well-established underlying models). For the turbine blades and towers, elastic beam models capable of capturing large nonlinear deformations (e.g., geometrically exact beam theory) will be sufficient for plant-scale simulations, where the models adequately incorporate detailed structure physics (e.g., complex laminates and internal structure).

\subsubsection{Numerical discretization}

For incompressible turbulent flow, there are multiple proven, mature discretization methods. In choosing one of these methods, we are guided by the need to leverage open-source software that is well supported, is demonstrated as a scalable HPC tool, and, in itself, leverages assets. For example, a linear-system solver should not be embedded in the discretization library, but should be itself an independent library. For flow-field spatial discretization, low-order unstructured grids are well established, and will be sufficient when combined with adaptive h-refinement. In regard to adaptive h-refinement, the known physics guide refinement policy; we anticipate refinement will result in a refined volume that encapsulates the meandering wake. Discretization approaches are required to have low numerical dissipation. Cell-centered and vertex-based finite-volume (FV) as well as continuous-Galerkin finite-element (FE) methods are viable candidates. Certain vertex-based FV and FE provide clearer paths (via p-refinement) to higher-order discretization than cell-centered FV. In the time domain, semi-implicit second-order discretization will be employed. In advancing a time step in this approach, the dominant computational cost is in calculating the pressure Poisson linear-system solution. This solve is the most significant barrier to scalability, and it is imperative that state-of-the-art solvers are leveraged. For the turbine structure well-established finite-element methods will be employed. As with the fluid-related 
software, turbine-structure software must be suited well for HPC and interaction with the flow solver suite.

\subsubsection{Meso-scale/micro-scale coupling}

Discussions and recommendations focused on the wind plant domain (micro-scale). The recommendations here were largely based on the concept of one-way coupling, where the mesoscale solution is taken as known and is driving the micro-scale. In particular, appropriate methods for turbulence "spin-up" remain an open topic that is covered in more detail in the Wind Plant Physics meeting. Whether or not two-way coupling is an important feature remains an open topic. There also remain issues surrounding the coupling of the micro-scale to numerical weather prediction and ABL codes.

\subsubsection{Fluid-structure coupling}

Limited discussion was devoted to fluid-structure coupling. However, for wind-plant-scale calculations, we envision that well-established loose-coupling (i.e. operator split) schemes and software will be leveraged.

\subsubsection{Decision-process overview}

The session panel was composed of experts spanning multiple disciplines in the computational fluid dynamics. Participants represented universities, national laboratories, industry, and the Army. Enthusiastic discussion was devoted to nearly all of the topics in the recommendations listed above. The following topics were debated extensively:

- Fully compressible and incompressible flow models

- Detached eddy simulation: zonal and seamless approaches

- Fully implicit and semi-implicit time integration

- Structured and unstructured meshes

- Finite-element and finite-volume methods

- Blade resolved, actuator line, and immersed boundary methods

- Adaptive mesh refinement strategies

- Sliding/body-conforming and overset meshing

- Low-order and high-order spatial discretization

- Role of numerical dissipation

- Complex terrain

The recommendations above were not necessarily the first choices for all participants, but they constitute a path forward considered viable by all.

\subsection{Addressing Software Engineering, Performance, and Scalability Issues on the Road To Exascale}

\section{Chair: Kevin Barker (PNNL) Co-Chair: Pat McCormick (LANL)}

\subsubsection{Description and summary}

Constructing a community simulation code designed to bridge disparate time and length scales presents a number of software engineering and design challenges. Add to this the requirement to scale to future exascale-sized systems and the challenges become even more daunting. This breakout session aimed to identify these challenges, as well as to begin to formulate strategies to address them. Topics covered included elevating performance prediction and modeling to a "first-class citizen" of the software-design process, software-design decisions that will impact 
scalability to possibly billion-way fine-grained parallelism, and potential strategies to address the challenges posed by advanced architectures.

It is generally accepted that a high-fidelity model of a wind plant will require exascale class computing. At the same time, the class of computing has the potential to enable a more fundamental understanding of the physics of wind plants and enable improvements that will increase the efficiency and lower the overall cost of energy produced by wind plants. True exascale computing remains several years in the future, but we know now that these computers will involve new architectural elements and will require new algorithms and codes to be used effectively.

From the perspective of the engineer, the entire workflow must also be considered, and again there will be significant changes driven by the architectures of exascale computers. High-level components of the workflow include

1. Pre-processing, including setting up the problem and mesh creation

2. Wind plant simulation

3. Post-processing and analytics

4. Uncertainty quantification and sensitivity analysis

5. Verification and validation

While the performance of these components can be considered separately, the scale of simulations that will be done will require that many, if not all, of these components be integrated into a single simulation.

Another goal of the A2E High-fidelity Modeling program is to create an open infrastructure to facilitate a modeling community and the use of exascale class simulation capabilities.

This breakout session developed a set of challenges that must be addressed if the potential of exascale computing is to be realized. These include the following:

1. Full workflow performance to improve overall productivity,

2. Software engineering for performance,

3. Code abstractions and libraries,

4. Performance characterization and optimization of simulation codes,

5. Architectures and infrastructure for coupling.

Each of these is described in more detail below.

It will be important that the A2E program engage broadly across the many communities that are targeting exascale computing to meet their simulation needs. Certainly there will be a need to leverage the many tools and infrastructure components that will be developed and to get early access to computers that include the architectural advances on the path to exascale computing. It will also be important to work across DOE programs and facilities, including the Leadership Class Computing Facilities, ASCR's SciDAC program, DOE's ACTT program and NNSA's ASC program.

\subsubsection{Full workflow performance to improve overall productivity}

Workflow performance must be optimized in order to enable increased productivity of wind energy researchers and users to reach technical decisions.

In complex, multi-faceted applications like this one, simulations that require high-performance computing - eventually exascale - are an important part of the overall workflow needed to yield insights and results that lead to decisions but not the only one. For example, if analysis of the output of the "grand challenge" simulations takes much longer to perform or cannot be done at all due to its scale, the ModSim project will not be able to meet its goals. Achieving good 
performance will require optimization over a wide parameter space. In the context of workflows, the metric for performance is productivity of the team that develops and uses it. Individual components have more traditional metrics such as scalability, efficiency, wall-clock time; those performance issues are treated in subsequent sections of this report.

Achieving high performance of the full workflow requires meeting many challenges. Components of the overall workflow execute on different systems. Software that requires little or no manual intervention to move data from one system to another will enable better productivity. At present it can take multiple re-tries and babysitting to move large files between systems. In addition, most of the components currently in use, such as mesh generation and visualization, were designed and implemented independently and will require modifications to enable their integration into a workflow.

Among the issues are the legacy formats of data, input and output, that are incompatible. Some of the components were not designed or implemented with scalability in mind and will become bottlenecks in the overall workflow unless they are re-implemented. In some cases there are few scalable software tools and collaboration with research projects in those fields may be needed to develop suitable components.

Training on performance issues will be needed. The developer community has uneven knowledge of approaches that lead to high performance of different algorithms on a variety of systems. The evolution of HPC computer architectures in the next decade will pose new performance issues and challenges, not only for the leadership-class systems but also mid-size clusters because they will often be scaled down versions of the same architecture.

The ModSim project will need multiple workflows. Some investigations will involve a handful of "hero" runs, jobs that use a large fraction of the largest systems for weeks. Others will require integrated analysis for the V\&V cycle, including many runs of moderate size jobs and statistical analyses of the outputs to yield confidence measures, for example, methods to evaluate trust in wind farm analysis.

Research, development, and widespread availability of tools for measurement and evaluation of workflow performance and productivity will be needed, in addition to the more traditional performance measurement tools that are discussed below. The R\&D should be conducted in collaboration with computer scientists and mathematicians who work in these domains; the ModSim project cannot undertake to develop every tool it needs and in some cases existing tools will provide a suitable foundation for what ModSim needs. In collaboration with computer scientists, the particle accelerator community has developed workflow approaches and software that might serve as models for what ModSim implements.

To achieve a common vision and practice, the project will define metrics for productivity, set up a clearinghouse for tested tools and documentation and case studies of performance characterization and optimization.

\subsubsection{Software engineering for performance}

This breakout session tackled the code performance aspects of software engineering as part of building the overall productive software development environment. Breakout 4 on "Infrastructure, Integration, and Software Development Processes" dealt with the challenges associated with collaborative development of a large complex software product with a geographically dispersed, multi-institutional team.

Software engineering for performance raises additional challenges, including

- Approaches for ensuring long-term performance awareness (e.g. part of regressions) 
- Finding the appropriate balance between architecture performance and abstractions, i.e., between ease-of-use and performance, and

- Design and implementation of coupling and library interface(s), since performance is often negatively affected in component coupling.

To avoid starting with a blank slate, ModSim will include legacy components into its software tree. Those components will often have poor performance because they use algorithms that were suitable for now outdated architectures, use data structures that lead to inefficient computation and I/O. Criteria need to be developed for including such legacy components despite their poor performance and plans for improving their performance or replacing them with new code.

Identifying and using "best practices" is expected to provide approaches and tools that will address those challenges adequately. There are various widely used tools for automated testing and regression that can be used to build the necessary infrastructure, including the addition of performance regression testing. Research is unlikely to be needed.

Because the ModSim software will be component based with some type of couplers, the regression testing has to go beyond component testing and include test harnesses for coupled components, with performance measurements.

There are now two dominant high-level computer architectures: many-core and CPU/GPU, aka accelerator based or hybrid, and these architectures will continue for the next decade, into the exascale regime. Therefore, software engineering for performance will need to deal with performance portability issues. How to achieve code portability is not well understood even in today's systems. Performance portability poses additional challenges. Regression testing on exemplars of each architecture will be needed but does not contribute to portability if there are separate code trees for each target system. Other sections of this report will address approaches for tackling performance portability.

\subsubsection{Code abstractions and libraries}

With the increasing number and complexity of HPC architectures and the lack of a standard programming model, it becomes critical to develop abstraction layers so that code developers are insulated from the details of the machine architecture. However this comes at a cost and often results in a decrease in performance. Abstraction comes at a cost.

Abstractions will also be a key element in enabling the integration of the full workflow, including pre-processing, post-processing, uncertainty quantification and optimization. In addition to integration, there will be a need for computational steering, i.e., changing the fundamental parameters of a simulation based on information and analytics computed while a simulation is in process.

This cost can be mitigated somewhat by the development of toolkits and libraries that implement commonly used functionality efficiently for each architecture. Examples of functionality that might be captured in a library include the following:

1. Linear and nonlinear solvers with common preconditioners

2. Time integrators

3. Input and output

4. Mesh operations, including refinement

5. Discretization templates and element libraries

6. Dynamic load balancing

7. Checkpointing and restart

8. Optimization and uncertainty quantification 
Even within a library, it may be possible to provide an abstraction for many of the architectural details through the use of templates and interface layers instantiated by libraries such as Kokkos.

Another goal is to provide as much flexibility as possible, both in the programming and in the selection of algorithms, deferring many decisions to compile time (in the case of a machine architecture), or even to run time (in the case of heterogeneous node architectures or dynamic selection of discretizations or solvers).

Certainly there is a lot of existing work across DOE and DOD laboratories and many academic institutions to develop abstractions and libraries targeted at exascale computers. It will be important to develop collaborations with these institutions and to leverage as much of the existing work as possible.

The ultimate goal is to leverage existing research and to achieve portability of the codes, including the portability of "good" performance, across a wide range of architectures, without putting the burden of performance on the wind plant modeler.

\subsubsection{Performance characterization and optimization of simulation codes}

It has always been challenging to extract code and workflow performance from large-scale computers. For the past two decades, the dominant HPC architecture has been relatively large, cache-based nodes with distributed memories and message passing based on MPI. However, this basic architecture will continue to evolve to include many-core nodes, vector and GPU accelerators, shared memories, and relatively slower communication between nodes. Significant research will be required to use these new architectures and tools to characterize performance, predict performance and diagnose performance problems will be critical. Furthermore, while we have a general idea of the basic architecture of an exascale-class machine, much of the application work must be done in advance of the availability of actual hardware.

The key tools for characterizing and optimizing performance on existing hardware are profilers and debuggers. These are generally machine specific and are often provided by the vendor. However, they are often ineffective for extreme-scale parallelism, they are not able to peel back the layers of abstraction to provide meaningful data to developers, and they are not predictive of performance on even closely related architectures.

Performance characterization and optimization are also becoming a more difficult and require more specialized knowledge and training. It is not reasonable to expect the wind plant engineers and model developers to develop these skills. It will be important to provide programming requirements, to work through cross-disciplinary teams, and to support people specifically trained in performance modeling.

Many decisions regarding performance must be made during the code-design process. These decisions include the basic code architecture, data structures and algorithms. However, it is also important that the code be designed for agility and for changes that are necessitated by changing architectures. It will also be important that performance testing, coupled with the usual regression, unit and system testing, be an integral part of the tools provided.

\subsubsection{Architectures and infrastructure for coupling.}

A very important component for wind-plant modeling is the ability to couple across both different physics and a wide range of scales. A monolithic code is impractical for many reasons, which will necessitate a code infrastructure for coupling codes and models into an integrated simulation on exascale platforms. From a software engineering standpoint, the focus must be on designing a programming interface and community standards for coupling while enabling the use of tools and infrastructure designed for high performance. There are examples of coupling infrastructures in 
various communities, including climate and nuclear energy. While these can provide an effective starting point, ultimately the coupling infrastructure will be driven by the specific use cases and modeling problems identified in the second A2E High-fidelity Modeling workshop.

\subsection{Infrastructure, Integration, and Software Development Processes}

Chair: John Turner (ORNL) Co-Chair: Richard Kendall (DoD HPCMC)

\subsubsection{Description and summary}

Participants in breakout group 4 included representatives of several projects and programs of scale similar to A2e and with collaborative computational science software development activities:

- ACME and CESM - the DOE and NSF climate modeling efforts

- ASCEM - the DOE/NNSA Advanced Simulation Capability for Environmental Management program for subsurface flow and transport in porous media

- CASL - the Consortium for Advanced Simulation of Light-Water Reactors, focused on advanced modeling and simulation for nuclear energy and managed by DOE/NE

- CREATE - the DOD/HPC Computational Research and Engineering Acquisition Tools and Environments program

- WISDEM - the DOE/EERE Wind plant Integrated System Design and Engineering Model

After introductions and initial discussion of common challenges, the group identified the following primary areas for attention:

1. Requirements

2. Developer workflow, including Testing

3. Software architecture

4. Component integration

5. Deployment and support

6. Infrastructure resources

For each area we then determined Requirements, Challenges, Options, and Recommendations. We list these aspects in the following sections. Although Testing is a subtopic within Developer Workflow, we considered it to be of sufficient importance to warrant its own section.

\subsubsection{Requirements}

For similar modeling and simulation programs, a progression of tangible, well-defined, prioritized use cases and associated simulation plans has been used to drive capability development. Documentation of these problems forms the requirements from which design and implementation plans can be derived.

This approach requires close collaboration with domain experts who understand the full system and can help develop a sequence of problems that will remain relevant over the life of the program.

A challenge with this approach is obtaining agreement among stakeholders on priority of problems. Cost models can be used to determine highest payoff problems, and the Board and/or advisory council(s) should accept and prioritize requirements

Recommendation 
Prior to development, based on interaction with stakeholders, develop a progression of specific use cases to be shared across the program.

\subsubsection{Developer workflow}

Developer workflow is the set of processes and tools members of the project team use to create software, whether tools for internal-project use or products for deployment to analysts. It should be flexible and adaptable, able to evolve to suit circumstances, including resources and experience of the team, stability of requirements, etc.

Note that there is a management overhead inherent in maintaining coordination and communication, and a balance between imposed uniformity of a centralized program and local autonomy can be challenging to maintain. In addition, efficiency due to consolidation must be balanced against DOE goals of multi-institutional participation.

\section{Recommendations}

- Review lessons learned from other govt. sponsored software development projects (WRF, CASL, CREATE, ASCEM, CCM, OPENMDAO, etc.).

- Survey "best practices" for scientific code development and leverage existing software and processes where possible in order to avoid having to create everything from scratch.

- Spend time up-front as a team selecting process and tools - vigorous documented debate of pros and cons.

- Spend time training developers as a team.

- Be aware of institutional barriers to communication and collaboration - can affect tool selection.

See the table later in this section for an overview of some input from other programs of similar size and scope to A2e.

\subsubsection{Testing}

A comprehensive hierarchy of tests (unit, regression, nightly, weekly, verification, validation) that ensures stability of the code base and confidence in results must be created as capability is developed, and must evolve in parallel. Ideally, a test system is integrated into the normal developer workflow in order to encourage use. Standard, widely-used tools such as CTest and CDash, along with new tools such as TriBITS, can be leveraged.

In some cases, defining and testing for success/failure can be a challenge. Care must be taken when comparison to "golden" output / results (previously deemed "correct") is used, particularly when using a fuzzy numerical difference tool to determine which changes are significant. Although small changes are to be expected due to changes in algorithms, parallel implementation, or even compilers and system software, it can be difficult to differentiate between benign changes and bugs.

Another challenge for a program like A2e will be use of legacy components with inadequate test suites. Resources must be expended to develop tests for these components that at least cover the capabilities required by the program.

\section{Recommendations}

- Clearly define level of testing that is considered adequate for the project and enforce.

- Develop a hierarchy of tests (some of which will only be possible on large systems) so as to minimize impact on developer workflow.

- Leverage tools that help with reporting (e.g., CTest, CDash).

- Include runtime and memory usage metrics in testing if possible. 
- Include testing for memory leaks (e.g., valgrind).

- Ensure that adequate hardware resources are available for testing.

\subsubsection{Software architecture}

A single overall software architecture can be difficult to define for programs as large and diverse as A2e, since it is unlikely that A2e will develop a single monolithic framework or application. A collection of components with shared infrastructure (such as the Virtual Environment for Reactor Applications, VERA, developed in CASL) will be a more likely path.

Some requirements and prerequisites when developing a modern computational physics environment include:

- Identify target platforms and compilers and language options as starting point. Support for additional platforms and compilers can be added as needed.

- Identify coupling approach (e.g., tight coupling, file based coupling, multi-executable, single executable).

- Define programming model(s) to be supported. These will most likely be MPI $+\mathrm{X}$, where $\mathrm{X}$ is pthreads, CUDA, OpenMP, OpenCL, OpenACC, etc.

- Identify target coupling architecture.

- Identify whether bit-for-bit reproducibility on different processor counts, run repeats and restarts are required. Obtaining bit-level reproducibility can be unduly restrictive.

- Identify whether components should share an I/O infrastructure and if parallel I/O should be required for each component.

- Identify which I/O formats will be supported (e.g., binary, netCDF).

- Standardize parameter input to be run-time accessible from input files.

- All component codes should be re-entrant.

Recommendations

- Empower chief architect to manage development team and decisions.

- Clearly define and document scalability, performance, and portability requirements.

- Spend time up-front as a team selecting architecture, programming model, and coupling approach - vigorous documented debate of pros and cons is healthy.

\subsubsection{Component integration}

We assume that both new and legacy modules, some of which may be proprietary, will be combined within the A2e environment. In addition to the technical challenges inherent in combining software that has never been combined before, and may not have been designed for integration, care must also be taken regarding ownership and licensing of integrated software products. Incompatible licenses may preclude use of otherwise attractive software.

Some potential challenges when working with legacy software include:

- Lack of modern data structures \& parallel programming algorithms

- Lack of vectorization and/or threading

- Lack of scalable memory and parallel I/O

- Non-standard software practices \& documentation

- Complex build systems based on custom scripts (rather than standard tools), causing portability challenges and difficult machine dependencies

- Non-standard, inflexible interfaces

- Unclear pedigree, ownership, and licensing 


\section{Recommendations}

- Establish a set of standards for software quality, documentation, performance, etc. for components to be integrated, and do not accept code not meeting standards - be prepared to fund improvement of essential existing components that do not meet standards.

- Maintain a set of APIs and require contributors to adapt their code to them or negotiate modification of APIs when necessary.

- Define an integration process, including a review process to vet software quality and scientific validity of candidate components.

- Require tests that ensure that code results obtained within the ModSim environment match standalone results.

\subsubsection{Deployment and support}

Some software that is developed is only used by the developers. In those cases issues such as licensing, documentation, and support are relatively unimportant. However, for programs such as A2e that develop and deploy software tools to be used by a broad developer and user community, those aspects of deployment become significant.

Licensing and background IP issues can be extremely complex for a multi-institutional project like A2e, particularly when leveraging existing software. It appears that A2e will emphasize open source licensing, which will help, but the differences between open source licenses must be understood by the team, since some are incompatible or impose undue restrictions when combined.

Documentation upkeep can be challenging from a funding perspective, since from a programmatic perspective capability development always appears more "productive" than development of documentation or training materials. Some projects address this by adopting a crowd-sourcing approach, or by setting aside a defined period (e.g. one day per week) as "documentation day."

\section{Recommendations}

- Use a license that allows the software to be freely distributable and modifiable.

- Provide a developer/user wiki, verbose code documentation. Provide regular user training courses. Keep documentation/courses up-to-date. Provide build instructions.

- Source should always available via popular version control systems. Strive for regular source/binary release dates.

- Provide a modular user environment for model input development, simulation launch (local, remote), and post-simulation data viz and analysis.

- Identify user community and define workflow (input, output, etc.) needs.

- Ensure standard communication interfaces between legacy and new components.

- Review lessons learned from other major open-source software development.

- Nightly builds pushed to user download site to enforce continuous integration.

\subsubsection{Infrastructure resources}

A core team devoted to the ModSim infrastructure will be critical to the success of A2e. This team will be focused on the software infrastructure and developer workflow - managing multiple repositories, build / test system, etc. Based on experiences of similar programs, the resources required for these activities are often underestimated. It can also be challenging to find staff with the necessary expertise due to the fact that staff with these skills are often undervalued at national labs and highly valued in industry (Google, etc.). 


\section{Recommendations}

- Dedicated hardware at lead institution to serve as reference software environment.

- Dedicated hardware at lead institution for regular testing.

- Infrastructure team dedicated full-time to A2e to as great an extent as possible.

- Core infrastructure team should be collocated if possible.

- Clearly identify HPC systems and allocation process.

- Stable long-term funding.

\begin{tabular}{|c|c|c|c|c|c|c|}
\hline & $\begin{array}{l}\text { Require- } \\
\text { ments }\end{array}$ & $\begin{array}{l}\text { Change } \\
\text { control } \\
\text { system }\end{array}$ & $\begin{array}{l}\text { Project } \\
\text { Mgmt } \\
\text { Tools }\end{array}$ & $\begin{array}{l}\text { Build } \\
\text { system }\end{array}$ & Test system & $\begin{array}{l}\text { Development } \\
\text { Philosophy }\end{array}$ \\
\hline $\begin{array}{l}\text { ACME / CESM } \\
\text { (Climate) }\end{array}$ & $\begin{array}{l}\text { CESM: } \\
\text { Community } \\
\text { Science } \\
\text { driven } \\
\\
\text { ACME: DOE } \\
\text { Science } \\
\text { driven }\end{array}$ & $\begin{array}{l}\text { CESM: } \\
\text { SVN/git } \\
\text { ACME: git }\end{array}$ & $\begin{array}{l}\text { ACME: } \\
\text { Confluence } \\
\text { + JIRA } \\
\text { CESM: } \\
\text { custom }\end{array}$ & $\begin{array}{l}\text { custom + } \\
\text { CMake }\end{array}$ & $\begin{array}{l}\text { custom + } \\
\text { CMake } \\
\text { ACME: Jenkins }\end{array}$ & $\begin{array}{l}\text { Continuous } \\
\text { integration for } \\
\text { BFB changes. } \\
\text { Custom - due to } \\
\text { chaotic system } \\
\text { and scientists } \\
\text { required to } \\
\text { distinguish } \\
\text { between roundoff } \\
\text { and climate } \\
\text { changing }\end{array}$ \\
\hline $\begin{array}{l}\text { ASCEM [1] } \\
\text { (Advanced } \\
\text { Simulation } \\
\text { Capability for } \\
\text { Environmental } \\
\text { Management) } \\
\text { Subsurface } \\
\text { Flow and } \\
\text { Transport in } \\
\text { Porous Media }\end{array}$ & $\begin{array}{l}\text { Use case } \\
\text { driven; } \\
\text { milestones for } \\
\text { code } \\
\text { development } \\
\text { driven by } \\
\text { meeting } \\
\text { milestones to } \\
\text { complete use } \\
\text { case } \\
\text { calculations. }\end{array}$ & $\begin{array}{l}\text { git, Trac, } \\
\text { mercurial, } \\
\text { sub- } \\
\text { version }\end{array}$ & Trac & CMake & $\begin{array}{l}\text { custom } \\
\text { (Python-driven } \\
\text { test suite, diff) }\end{array}$ & $\begin{array}{l}\text { modular, major } \\
\text { components } \\
\text { utilize well } \\
\text { defined } \\
\text { communication } \\
\text { interfaces }\end{array}$ \\
\hline $\begin{array}{l}\text { CASL [2] } \\
\text { (Consortium for } \\
\text { Advanced } \\
\text { Simulation of } \\
\text { Light-water } \\
\text { Reactors) }\end{array}$ & $\begin{array}{l}\text { Challenge } \\
\text { Problems - } \\
\text { Industry } \\
\text { Partners }\end{array}$ & git & $\begin{array}{l}\text { Trac (heavily } \\
\text { customized) } \\
\text { for phase 1, } \\
\text { evaluating } \\
\text { alternatives } \\
\text { (Jira or } \\
\text { Fogbugz) for } \\
\text { phase } 2\end{array}$ & $\begin{array}{l}\text { CMake + } \\
\text { TriBITS }\end{array}$ & $\begin{array}{l}\text { CTest, CDash, } \\
\text { "golden" results } \\
+ \text { diff, pre- } \\
\text { push, post- } \\
\text { push, nightly, } \\
\text { and weekly, } \\
\text { valgrind } \\
\text { periodically }\end{array}$ & $\begin{array}{l}\text { Kanban, near- } \\
\text { continuous } \\
\text { integration }\end{array}$ \\
\hline $\begin{array}{l}\text { CREATE } \\
\text { (Computational } \\
\text { Research and } \\
\text { Engineering } \\
\text { Acquisition } \\
\text { Tools and }\end{array}$ & $\begin{array}{l}\text { Stakeholder } \\
\text { defined use } \\
\text { cases }\end{array}$ & SVN/git & $\begin{array}{l}\text { JIRA / } \\
\text { Confluence }\end{array}$ & $\begin{array}{l}\text { CMake / } \\
\text { Jenkins (for } \\
\text { continuous } \\
\text { integration) }\end{array}$ & $\begin{array}{l}\text { CTest/Boost, } \\
\text { diff \& "gold" } \\
\text { files }\end{array}$ & $\begin{array}{l}\text { modular / test } \\
\text { driven / } \\
\text { continuous } \\
\text { integration }\end{array}$ \\
\hline
\end{tabular}




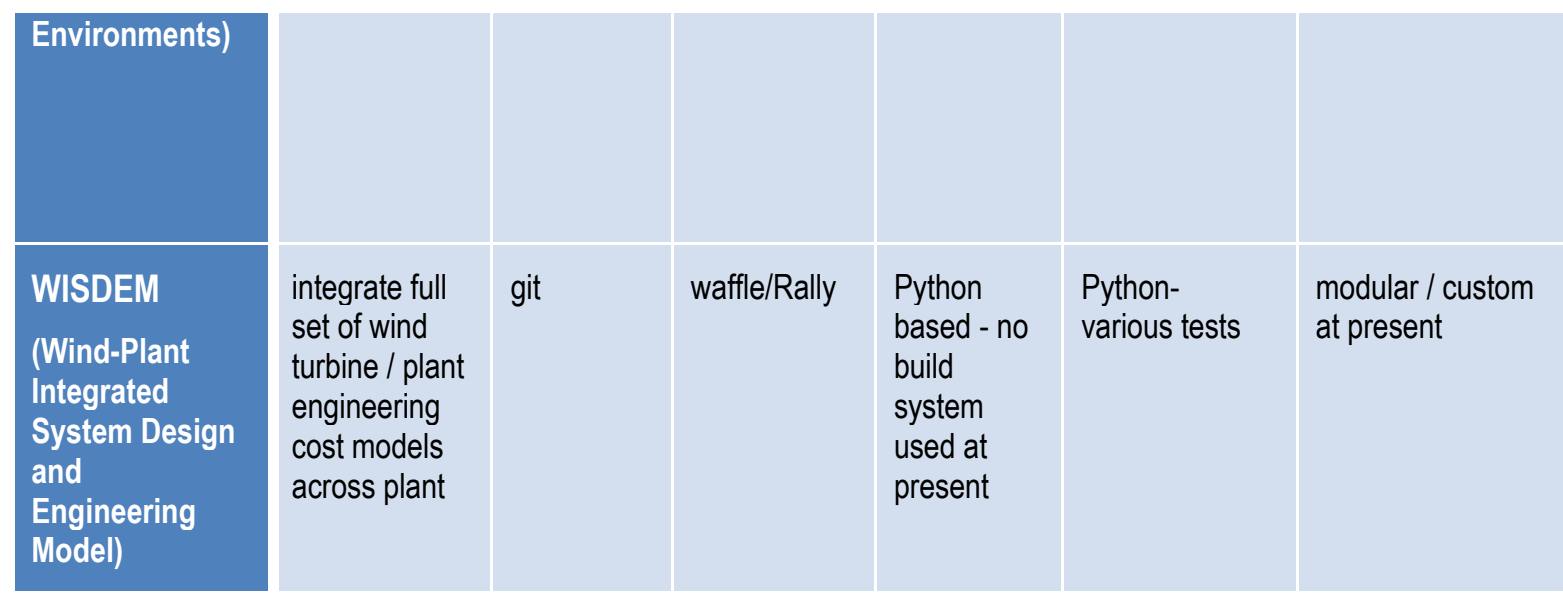

Notes:

[1] Encourage that design decisions by computational science and computer science team are informed by domain experts experience in relevant field to ensure the numerical pitfalls of 10-20 years ago are not re-discovered by the current development team.

[2] In CASL, a Board of Directors provides high-level guidance and recommendations, and Industry and Science Councils provide technical feedback. It is important to have these two Councils interact with each other.

A set of survey questions was proposed to help gather further lessons learned from programs similar in size and scope to A2e.

Requirements:

- Who defined the overarching requirements?

- How much was prescribed versus how much was up to the team?

- How much interaction with stakeholders was done and how was that organized?

Developer Workflow:

- In spectrum from agile to milestone drive development, what choices were made and how does your project handle workflow management?

- How did you make the decision? What influenced your approach?

- What tools do you use to support the process?

- How do you handle legacy codes in that process as well as working with institutions that may use incompatible software management tools or processes?

Software Architecture:

- Describe general process by which software architecture was defined;

- What were the key decisions that were made and how was that managed?

- Have there been any major architectural changes since the project began?

- How did you incorporate the various perspectives from computer, domain and computational scientists?

Component Integration Process:

- Do you deal with legacy code integration and what have been the biggest challenges to that process?

- Do you deal with code contribution from third-parties and what have been the biggest challenges to that process? 


\section{Deployment and Support:}

- Who are the users of your software?

- What is the level of sophistication from your users on the code?

- What kind of training do your provide?

- Support?

- What software tools (if any) do you use for support?

- For licensing, do you deal with open-source licensing? If so, what types?

Infrastructure Resources:

- What is a rough estimate for annual budget and FTE's for the project?

- What are the supporting IT resources used in terms of organizational IT personnel, computing resources, etc? 


\section{Wind Plant Physics and Modeling}

\subsection{Meeting Overview and Primary Outcomes}

The Wind Plant Physics and Modeling meeting focused on defining requirements for state of the art, high-fidelity modeling and simulation of the flow through entire wind plants and resultant wind turbine aerodynamic loads. Attendees included a diverse mixture of modelers, experimentalists, and wind plant technology experts from universities, national laboratories, and industry. The attendees were tasked with identifying and prioritizing key physics modeling challenges, defining the validation data needs to address these challenges, and identifying requirements for the simulation software from the physics modeling and validation perspectives. In order to focus the discussions, the following three "Use Cases" were provided to the attendees ahead of the meeting.

1. Understand and Discover: Use a HFM, in conjunction with sparse field data (data assimilation), to perform a forensic analysis. For example, how do specific weather events and/or atmospheric turbulence cause a particular transient load on one or more turbines within a wind plant?

2. Predict and Evaluate: Use a HFM to establish a wind farm power curve, and apply the model to selected new technologies and operating strategies to assess the impact on plant power output.

3. Design and Control: Use a HFM, with integrated uncertainty quantification methods, to develop reduced order models that can be used for design and control of wind plants.

The desired simulation capabilities must span a huge range of scales, from the meteorological mesoscale $\left(10^{4}-10^{5}\right.$ meters $\left.(\mathrm{m})\right)$ and wind plant scales $\left(10^{3}-10^{4} \mathrm{~m}\right)$, to the atmospheric boundary layer microscale $\left(10^{2}-10^{3} \mathrm{~m}\right)$ and the turbine rotor scale $\left(10^{2} \mathrm{~m}\right)$, to the blade chord $\left(10^{0} \mathrm{~m}\right)$ and blade boundary layer $\left(10^{-3} \mathrm{~m}\right)$ scales. The two-day meeting was organized around group breakout sessions that addressed not only the major categories of scales - mesoscale, plant scale, turbine scale - but also the interfaces between scales. Intentional organization of breakouts along scale interface allowed for cross-cutting discussions that broke through traditional barriers within the wind plant modeling community. The first day of the meeting was structured around two breakout sessions, each of which was tasked with identifying and prioritizing physical phenomena that represent key modeling challenges for HFM. The PIRT table construct was used to facilitate and organize output from these sessions (see 3.2). On the second day, the groups again gathered by physical scale interface, and defined priority model validation data requirements as well as simulation software requirements from the perspective of the modeling community.

The breakout session charters were defined ahead of the meeting as follows:

\section{Breakout Session \#1: Phenomena Identification and Ranking by Scale}

Primary Goal: Complete a gap analysis for high fidelity modeling of wind plant physical phenomena within a specified range of physical scales.

The breakout groups will be given an initial Phenomenon Identification Ranking Table (PIRT), and asked to fill out this table and to make it as complete as possible. This process will involve cataloguing of the physical phenomena that must be captured by models in order to make the desired predictions at wind plant scale. The process also involves a prioritization of the phenomena, as well as an assessment of the current state of model adequacy. The groups should 
spend some time discussing the appropriate class of models and their resolution requirements for each of the phenomena.

\section{Breakout Session \#2: Phenomena Identification and Ranking by Scale Interface}

Primary Goal: Complete a gap analysis for high-fidelity modeling of wind plant physical phenomena important to the specified interface between physical scales.

The breakout groups will build on the work completed during breakout session \#1. Firstly, they will review the PIRT tables by scale, and make any modifications deemed necessary. They will then proceed to augment these PIRT's with PIRT table entries focused on the interface of two scales (e.g., mesoscale/wind plant scale). Additional modeling requirements and strategies should be identified that address the scale interface phenomena, and model requirements at the individual scales should be re-assessed in light of this new information.

\section{Breakout Session \#3: Validation Data Requirements}

Primary Goal: Identify and characterize existing, currently planned, and future experimental campaigns that must be performed in order to ensure an adequately validated high-fidelity wind plant model.

A2E will involve tightly integrated modeling and experimental measurement efforts. In this session, the breakout groups are charged with identifying specific data sets that will be needed to validate the high-fidelity models. Validation of a model for a complex system such as a wind plant will require a hierarchy of problems, from relatively simple single-physics problems to more complicated multi-phenomena sub-system problems, and finally the full system. The breakout groups should define specific requirements for experimental campaigns that will adequately address validation data needs for models across this entire heirarchy.

\section{Breakout Session \#4: ModSim Software and Environment Requirements}

Primary Goal: Define requirements for the high-fidelity simulation code(s), based on the need to properly represent and/or resolve the physical phenomena already identified.

The breakout groups will form requirements for the simulation codes, in terms of accuracy, robustness, and time-to-solution. Requirements for model interfaces, as well as individual models, should be defined. Additional requirements related to the complete simulation workflow, such as mesh generation, visualization, data mining, and other post-processing should also be considered. The recommendations from this breakout group should be made in the context of the use cases/challenge problems and physical phenomena that have been identified during previous breakouts.

A number of invited talks and plenary discussion sessions were interspersed between breakout sessions in order to frame the discussion and provide important "big picture" discussions to take place with participation from the entire group of attendees. These included opening remarks from Jose Zayas, Director of the DOE Wind Program, along with an overview of the A2E initiative from Mike Robinson, also from the DOE Wind Program. Rich Hills (SNL) discussed the meaning and structure of a "validation-directed" R\&D program, and outlined the PIRT process that was subsequently used by the breakout groups. Prior to breaking out by scale interface, Jeff Mirocha (LLNL) and Jim Brasseur (Penn State U.) gave a collaborative talk including contributions from other co-authors outlining the special challenges in modeling the interface between the atmospheric mesoscale and microscale. On Day 2, Greg Oxley (Vestas) and Philippe Beaucage (AWS Truepower) gave separate talks, each of which gave an industry perspective on how HFM is currently being applied within the wind industry and how each company views the future of HFM. These talks made it clear that the industry is relying on government entities to push the state-of-the-art in HFM, both in model development as well as next-generation 
software development. Prior to breaking out to discuss model validation requirements, David Maniaci (SNL) gave a collaborative talk summarizing current DOE wind plant data campaigns and describing DOE test facility and instrumentation assets that may provide opportunities for future validation data campaigns. Prior to breaking out to discuss simulation software requirements, Mike Sprague (NREL) gave an overview of the major outcomes from the ModSim Environments planning meeting.

The major outcomes from the Wind Plant Physics and Modeling planning meeting are summarized here.

1. Modeling the planetary boundary layer and providing accurate transfer of information across the mesoscale/microscale interface are critical research areas.

2. The current understanding of wake behavior within wind plants is in urgent need of improvement, and validated high-fidelity models are expected to address this gap. The behaviors identified with relatively low understanding and/or modeling gaps include wake generation, merging, meandering, skewing, and dissipation, as well as how the near wake influences plant aerodynamics

3. At the plant scale, significant gaps exist in modeling of the atmospheric boundary layer for complex terrain as well as modeling of surface conditions such as roughness, vegetation, heat flux, and soil moisture. These gaps must be addressed to allow for predictive high-fidelity wind plant simulations beyond canonical flat-terrain cases.

4. At the turbine and blade chord scales, complex vorticity fields are generated which interact with one another and with the atmospheric turbulence (and perhaps with turbulence from other wakes) to form the near wake. High-fidelity modeling is needed to address deficiencies in understanding and predicting the initial generation of this vorticity at each individual turbine, and in understanding how the near wake develops and influences plant scale aerodynamic behavior.

5. Validation data requirements for each physical scale were generated by the breakout groups. A common theme across all scales is that, depending on the particular phenomena being addressed, data may be needed at either full wind plant scale, at subscale field facilities, within controlled wind tunnel environments, or at some combination of these three experimental environments.

6. The major elements of simulation software requirements captured during the Modeling and Simulation Environment Planning Meeting were largely reaffirmed by the modeling community, while some additional useful detailed requirements related to workflow and target efficiency of the simulation environment were collected.

The following subsection describes the PIRT planning process that was used during the meeting breakouts. The subsequent subsections provide more detail on the outcomes of each breakout group.

\subsection{Role of the PIRT for A2e Planning}

The overall purpose of the Wind Plant Physics and Modeling planning meeting was to continue integrated planning of HFM software, model development, experimental, and other needs to ensure that the resulting HFMs are predictive, credible, and useful to the analyst as well as the industrial customer. Specific tasks for this meeting were to identify the important physical phenomena that should be simulated by the high-fidelity computational models, assess the capability of these models to represent this physics, to perform an assessment of model development, verification, validation needs, and to identify HFM software requirements from an analyst perspective. These assessments provide structured information to be used to directly support the planning of an integrated A2e HFM/Experimental program to meet the A2e program objectives. 
To expedite and document the discussions, the working groups were organized by scale and by cross-scale phenomena, with each group tasked to develop a corresponding Phenomenon Identification Ranking Table (PIRT). The PIRT is a spread-sheet, listing the phenomena identified as important by subject matter experts in the first column, followed by columns with assessments of the importance of the phenomena relative to the HFM predictive objectives (ranked H, M, L), our understanding and ability of the mathematical models to represent this phenomena, followed by an assessment of verification and validation evidence that the computational algorithms solve the mathematical models that represent this phenomena. This information is used to identify and characterize modeling and experimental data (including validation data) needs to help insure that the HFM tools will be predictive, credible, and useful. The PIRT is the most commonly used approach for characterizing computational model capability and model/validation needs from a physics perspective. This tool was first developed by the nuclear power industry, and has become the standard approach to addressing model capability needs from a planning perspective for complex, multi-physics engineered systems.

The role of the PIRT in integrated A2e HFM/Experimental program planning is illustrated in the top blue box of Figure 1 below. The PIRT, along with the identification of the validation needs across scales (i.e. across the validation hierarchy), forms the backbone of program planning. The resulting PIRTs, along with PIRTs developed by smaller A2e teams during Q1 of FY15, are currently being used to identify overall experimental program needs that are specifically designed to support HFM development and model validation for prediction of plant scale performance as well and turbine response.

More detailed experimental planning and validation effort, based on the results discussed above, are reflected by the second blue box of Figure 1. Such experimental planning has been initiated during FY15 for model validation experiments at both the wind tunnel scale and the DOE Scaled Wind Farm Technology (SWiFT) scale based on PIRT results. 


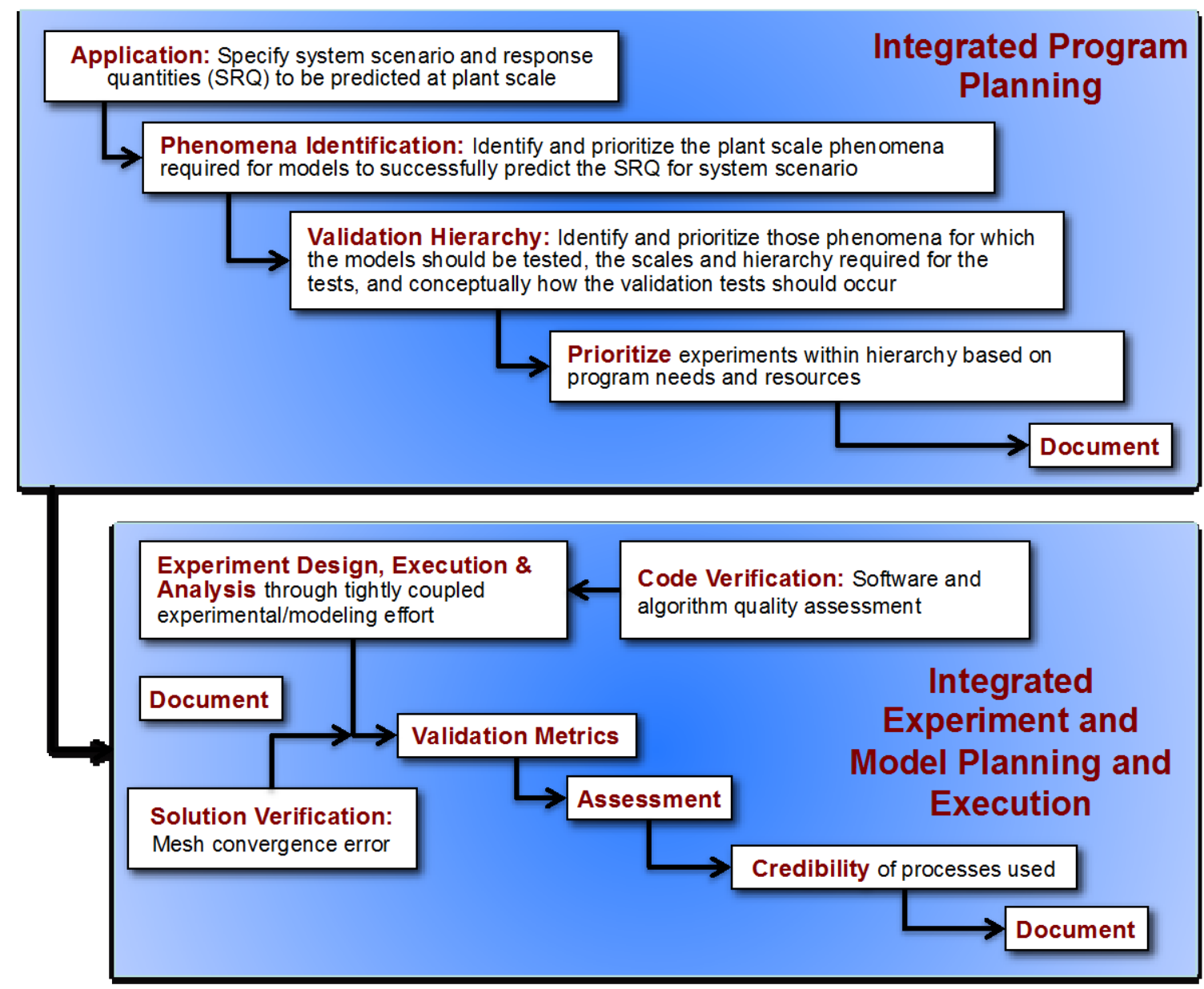

Figure 1. This figure illustrates the process used by the break out groups, following Phenomenon Identification Ranking Table (PIRT) to identify and rank phenomena of interest at the various wind plant scales and scale interfaces. 


\subsection{Breakout: Mesoscale and Mesoscale/Plant Scale Interface}

\section{Chairs: Cathy Finley (Wind Logies), Jeff Mirocha (LLNL), Larry Berg (PNNL), Pat Moriarty (NREL)}

\subsubsection{Physical Phenomena at the Mesoscale}

There are a large number of meteorological processes that are important for short-and long-term forecasts of wind distributions impacting power production. In the context of this document, the focus is on identifying and prioritizing key phenomena that have a large impact on the wind energy industry, as opposed to fundamental numerical weather prediction. In the context of the group's discussions, the following phenomena were identified:

1. Wind and turbulence in the planetary boundary layer (PBL)

2. Surface-atmosphere interaction (including both land and water)

3. Low level jets, including their morphology and structure

4. Terrain effects

5. Extreme wind events that impact plant operation and turbine design

6. Mesoscale horizontal pressure gradients

This list is quite extensive, requiring prioritization of those that are most relevant for the wind power industry. This rating is based on the expert opinion of the group and the items were selected because they are important on a daily basis, or have a large impact on the maintenance and operation of the wind plant. Other weather events that impact the wind plant may be associated with meteorological phenomena that are beyond the scope of this group, but it is still important to capture their cumulative impact on the wind speed and wind shear over the rotor diameter. For this reason we are not considering weather forecasting, but rather we are focused on the simulation of specific meteorological phenomena most relevant to physical processes within the PBL. Wind and Turbulence in the PBL: The most important phenomena are the distributions of wind speed and direction, temperature, and turbulence within the PBL. The PBL represents the operating environment of the wind plant. The current generation of mesoscale models has known deficiencies associated with the parameterization of the sub-surface, surface, and PBL that impact the winds at the rotor.

Surface-Atmosphere Interaction: Processes at the surface are a key driver of the boundary layer structure and evolution (including vertical profiles of winds, temperature, and turbulence). The representation of surface processes used for numerical weather prediction is not of sufficient fidelity (e.g. the application of Monin-Obukohv similarity theory) to accurately represent the lowaltitude wind profile. Likewise, the partitioning between sensible and latent heat fluxes, as well as poor representation of sub-grid variability of land-use and plant phenology strongly influences both the mean winds and turbulence at rotor heights.

Low Level Jets: Low level jets are the largest driver of the nocturnal wind resource over the central United States, and also lead to large amounts of wind shear over the rotor diameter. Breaking atmospheric waves, which are often associated with LLJs, can have a large impact on the lifespan of a wind turbine. While most prevalent over the Great Plains, LLJs can be found in many other locations, including along terrain features or as barrier jets near shore lines. The entire lifecycle of the LLJ, including its structure and morphology, are intimately tied to the representation of processes within the PBL as they interact with the mesoscale structure of the atmosphere.

Terrain Effects: There is continued development of wind plants in regions of complex terrain. It is important to note that even moderate variations in terrain can have significant impact on the mesoscale distribution of wind speed at rotor height. Terrain effects can generate unique flows 
with spatial scales ranging from small scale turbulence (e.g. flow separation), to gap flows, to wakes associated with terrain features, to mountain waves and can also vertically displace the mean flow leading to internal boundary layers around the wind plant.

Extreme Events and Mesoscale Pressure Gradients: Extreme events are frequently tied to large-scale weather features that fall within the domain of numerical weather prediction and as such are largely out of scope for this report. For a given mesoscale state, it is important that our current modeling tools represent the vertical and horizontal winds and turbulence over the depth of the PBL, especially at rotor heights. Likewise, the simulation of the horizontal pressure gradients is a function of the large-scale model and/or initialization and is an issue associated with numerical weather prediction.

\subsubsection{Physical Phenomena at the Mesoscale-Microscale Interface}

Mesoscale simulations alone only explicitly resolve boundary-layer structure due to the large spatial scales that must be used to capture the large-scale weather features. Therefore, the actual microscale flow structures (turbulence) that impact the rotor cannot be explicitly resolved on mesoscale simulation domains. The effects of microscale are instead parameterized in the mesoscale model. To accurately represent flow structures that impact the rotors requires accurate definition of both the mesoscale and microscale phenomena via coupling of the meso- and microscale simulation methodologies

In order to accurately couple these simulation methodologies the following issues were identified as high priority:

1. Passing relevant information from the mesoscale PBL to the microscale domain

2. Representation of surface layer

3. Profile of dynamics and thermodynamics

4. Parameterization appropriate for high resolution mesoscale simulations

Passing Information from Mesoscale to Microscale: Specification of mesoscale inflow properties can provide information required for the development of microscale features consistent with the larger-scale forcing. Winds, temperature, humidity, and other mesoscale turbulence parameters (e.g. TKE, stresses, PBL height), as functions of space and time, must be projected onto the microscale model grid mesh. Because of similarity of the two topics, we have combined items 1 and 3 .

Representation of surface layer: High-fidelity treatment of the surface layer that is consistent with the resolution of the microscale model is required. Over land this includes the distribution of vegetation (including height and evapotranspiration) and surface characteristics. Over water this includes details of the waves (including wave spectra, wave age, and swell).

Parameterization appropriate for high resolution mesoscale simulations: Item 4 above describes the need for parameterizations of the mesoscale PBL information at higher horizontal resolution than has traditionally been utilized, using procedures that have not been properly validated at these scales. Application of mesoscale models at high resolution (less than approximately $10 \mathrm{~km}$ ) violates several assumptions upon which the traditional mesoscale parameterizations are based. This calls into question their applicability for high resolution mesoscale simulations, which therefore impacts mesoscale-microscale coupling. New parameterizations that would allow simulation along the spatial continuum would take significant theoretical development. Therefore we have focused on the application of contemporary highresolution mesoscale parameterizations (e.g. HRRR simulations at $3 \mathrm{~km}$ horizontal resolution). 


\subsubsection{Validation Data Requirements}

The data required for evaluating mesoscale simulations and the mesoscale-microscale coupling are similar, differing mainly in the horizontal spacing of the measurements. Accurate measurements of wind, temperature, and humidity are needed from the surface, including the soil moisture and surface flux layer, through the lowest several kilometers of the atmosphere. To the extent possible, measurement of turbulence and turbulence statistics are also needed. Before new measurement strategies are planned, efforts should be made to examine existing data sets or networks.

One important difference for the mesoscale and mesoscale-microscale coupling is the horizontal spacing of the measurements. By their nature, mesoscale simulations cover a large geographic area and measurements should be distributed through the simulation domain. In contrast, the mesoscale-microscale coupling occurs on a relatively small scale and requires measurements be made in closer proximity to one another.

In either case, there are science questions for which a reference facility or facilities are appropriate (which could be accommodated by a "mobile" facility). Reference facilities are marked by high quality measurements at high temporal scales that are conducted for a multi-year period of time. In order to meet the needs of the wind energy community reference facilities should be deployed in a number of different geographic locations, including areas of complex terrain or over open water. Key variables measured at the reference are the vertical profile of turbulence (e.g. Reynolds stresses, heat fluxes, integral length scale, dissipation rate, spatial correlations), temperature, and humidity with vertical resolution approaching $10 \mathrm{~m}$ (and perhaps finer spacing in the surface layer and coarser spacing above the rotor). These profiles should extend several kilometers above the surface. The reference site should also include measurements that capture the three-dimensional structure of the boundary layer and potential LLJs, which could be accomplished using scanning remote sensing system. The reference facility should be deployed with a distributed network of meteorological observations that would provide mesoscale sampling of a reduced set of key variables at a limited number of heights, such as surface fluxes, wind speed and direction, and turbulence. As needed, the distributed sites could also include profiling or scanning remote sensing instruments. It is worth noting that data collected by the reference facility and distributed network could also be assimilated into mesoscale or microscale models.

As we require high-fidelity surface interactions in the microscale simulations, additional measurements of relevant terrain and/or sea state features will be required. Over land, these variables include the land-use/land-cover and vegetation characteristics, including height and spatial variability. Over the large bodies of water, these variables include wave spectra, wave age, breaking waves, swell, and sea-surface temperature.

It is important that the data collected be stored in an archive that includes metadata and measurement uncertainty. 


\subsection{Breakout: Plant Scale and Plant Scale/Turbine Scale Interface}

\section{Chairs: Pat Moriarty (NREL), Greg Oxley (Vestas), Dominic von Terzi (General Electric)}

\subsubsection{Physical Phenomena}

Important phenomena at the plant and plant-to-turbine scales were identified that impact efficiency and reliability of wind plants. Only those considered to pose the most severe barriers to progress in HFM of wind plants are discussed in the following. As a result of the PIRT analysis, three primary phenomenological groups emerged:

- Atmospheric boundary layer (ABL)

○ Quasi-stationary phenomena based on atmospheric stability like shear, veer, stratification, low-level jets, roll cells, boundary layer height, vertical \& horizontal variations, geostrophic wind

- Surface conditions like roughness (including forests, canopy), heat flux \& moisture

○ Topography (including complex terrain \& man-made structures)

- Special events like bursts, Kelvin-Helmholtz waves, ramps, frontal passages, thunderstorms outflows, etc.

- Wakes

- Far wake phenomena like meandering, merging, skewing/asymmetry and dissipation

- Near/far wake transition

- Ability to control wakes (e.g. steering)

- Plant scale phenomena

- Acoustic propagation within the turbulent atmosphere and over complex terrain

- Large plant aerodynamics such as vertical momentum flux, deep array, and blockage effects

\subsubsection{Model Status and Gaps}

Initial studies on most phenomena related to both wakes and the ABL have already been carried out or could be initiated - however, clearly more work is needed. To ensure impact these studies would highly benefit from, or even critically depend on, progress towards both a formal validation program and a more efficient simulation environment. Both needs are discussed in more detail in the sections below.

In addition, there are significant physical modeling gaps in the following areas:

- $\mathrm{ABL}$ in complex terrain,

- $\quad$ surface conditions such as roughness, heat flux and moisture, for example; note that for moisture, we need to first establish the sensitivity and relevance,

- acoustic propagation.

Regarding wakes, all topics that were identified as having a high impact on farm performance were ranked equally low in maturity of understanding of the physical mechanisms and the ability to capture the effects in reduced order models. Progress is urgently needed here. Regarding ABL, two areas were in need of similarly urgent model development and validation: (1) shear and veer effects and (2) special atmospheric events. For the other topics, understanding and validation has already reached a medium state, but still needs further improvement. 


\subsubsection{Validation Data Requirements}

First, the availability of adequate validation data for high-priority physical phenomena was discussed. Those identified as "low" adequacy (see Table 1) are of grave concern for future highfidelity studies and hence need immediate attention.

In addition, the scale recommended for a given validation campaign was identified. For the plantscale phenomena group, full scale campaigns are needed. For wake investigations, it is strongly encouraged to design complementary campaigns involving all scales: full and reduced scale field experiment as well as reduced scale wind tunnel campaigns. Often there is a balance between testing cost, observable resolution and the scale of the test, so any physics that can be observed accurately at smaller scales than full scale should be encouraged. Studies that examine common phenonema across different scales (possibly in simulation environments) should also be done to determine applicability of smaller scale tests. Note that full-scale field experiments should first be for homogeneous terrain with largely homogeneous inflow to the farm. It is also important to emphasize the interactions between modeling and observational efforts must be strengthened for example, simulation should be used to design experiments and even help quantify the limitations and uncertainties of instrumentation, and validation of models must take place both during and after observational campaigns are executed.

For the ABL studies, very different needs emerged (seeTable 1) and several important phenomena are only observable at full scale, which are dependent on large scale atmospheric forcing. Specific data requirements are dependent on the phenomena to be observed, with specific instrumentation applying to certain phenomena and scale.

Table 1. Priority physical phenomena at the wind plant and plant/turbine scales with validation data requirements.

\begin{tabular}{|c|c|c|c|}
\hline $\begin{array}{l}\text { Physical } \\
\text { Phenomena }\end{array}$ & $\begin{array}{l}\text { Availability } \\
\text { of Adequate } \\
\text { Validation } \\
\text { Data }\end{array}$ & $\begin{array}{l}\text { Recommended Scale of } \\
\text { Experiments }\end{array}$ & $\begin{array}{l}\text { Validation Data } \\
\text { Requirements and } \\
\text { Considerations }\end{array}$ \\
\hline $\begin{array}{l}\text { Plant Scale } \\
\text { Phenomena }\end{array}$ & Low & Full scale & $\begin{array}{l}\text { Large array/internal } \\
\text { boundary layer effect, } \\
\text { vertical and lateral } \\
\text { momentum flux, measure } \\
\text { above and around wind } \\
\text { plants and possibly above } \\
\text { ABL height }\end{array}$ \\
\hline Wakes & Low & $\begin{array}{l}\text { Complementary } \\
\text { experiments at all scales }\end{array}$ & $\begin{array}{l}\text { Merging, meandering, } \\
\text { asymmetry, dissipation, near- } \\
\text { to-far transition, steering, all } \\
\text { dependent on atmospheric } \\
\text { conditions, observations of } \\
\text { wake centerline motion }\end{array}$ \\
\hline ABL -shear & Low & $\begin{array}{l}\text { Complementary } \\
\text { experiments at all scales }\end{array}$ & $\begin{array}{l}\text { Dependent on surface } \\
\text { roughness which is difficult } \\
\text { to quantify }\end{array}$ \\
\hline
\end{tabular}




\begin{tabular}{|l|l|l|l|}
\hline ABL - veer & Low & Full scale & $\begin{array}{l}\text { Highly dependent on } \\
\text { stability, so temperature } \\
\text { profiles and ABL height } \\
\text { measurements important }\end{array}$ \\
\hline $\begin{array}{l}\text { ABL - special } \\
\text { events }\end{array}$ & Low & Full scale & $\begin{array}{l}\text { K-H waves, turbulence } \\
\text { bursting, roll cells, } \\
\text { phenomena are intermittment } \\
\text { and dynamic }\end{array}$ \\
\hline $\begin{array}{l}\text { ABL }- \text { surface } \\
\text { flux }\end{array}$ & Low & $\begin{array}{l}\text { Complementary } \\
\text { experiments at all scales }\end{array}$ & $\begin{array}{l}\text { Heat and moisture } \\
\text { measurements have large } \\
\text { spatial inhomogeneity }\end{array}$ \\
\hline $\begin{array}{l}\text { ABL }- \text { complex } \\
\text { topography }\end{array}$ & Low & Full scale & $\begin{array}{l}\text { More complexity requires } \\
\text { more instrumentation }\end{array}$ \\
\hline ABL - all other & Medium & Check for existing data & \\
\hline
\end{tabular}




\subsection{Breakout: Turbine Scale, Blade Chord Scale, and Turbine/Blade Chord Interface}

\section{Chairs: David Maniaci (SNL), Fotis Sotiropoulos (U. of Minnesota), Jon Naughton (U. of Wyoming), Jim Brasseur (Penn State U.)}

\subsubsection{Turbine Scale Phenomena}

The primary goal of the turbine scale breakout session was to complete a gap analysis for highfidelity modeling of wind plant physical phenomenon within the turbine scale. The gap analysis was accomplished by completing the relevant PIRT Table. The definition of the turbine scale was discussed at length, and many of the important phenomena of interest were identified during this discussion. It was decided that the turbine scale would be defined by the scale of turbine system components that create, affect, and react to the wind turbine wake, as well as by the time and length scales of flow phenomenon that compose and affect the wake. A summary list resulting from this discussion is given below, which correlates to the turbine scale phenomena in the PIRT created during this breakout session, which is shown in the Appendix.

\section{Phenomena that Create and Affect the Wake:}

- Inflow: due to the ABL and the wake(s) from upstream turbines

- Response of the wind turbine system to loads and conditions including:

○ Deformation of blades and tower

- Drivetrain feedback via shaft deflection and blade rotation rate and acceleration

- Control system response (sensors -> yaw, pitch, generator, brake)

- Interaction between the flow field around the rotor, tower, and nacelle, as well as the interaction with the resulting wake(s).

- The blade flow field, as it directly relates to the near wake.

- Interactions between wake phenomena and scales. The near wake transitions to an intermediate wake, which transitions to the far wake, which interfaces with the plant scale.

\section{Important Scales}

- The definition of the most important (or energetic) scales depends on the given modeling scenario, which is defined by the environment and quantities of interest.

- Wake Length Scales: from the tip vortex scale ( $\sim$ chord) to the rotor scale ( diameter)

- Time scale: Tip vortex advection rate to blade rotation rate

Much of the group's discussion came back to confusion over the difference between physical phenomena and how those phenomena are modeled. A proposal was made that a separate PIRT be developed for blade boundary layer resolved turbine simulations and for simulations that use the actuator line blade element method. It was eventually decided that the PIRT would cover both types of models, as the physical phenomena relevant to turbine scale physics do not depend on the type of model being used.

\section{Ranking}

The priority ranking was accomplished after the phenomenon identification period, which resulted in almost all of the turbine scale phenomena being listed as highly important. This was deemed acceptable by the group, as less important phenomenon that were discussed during the identification period were not added to the chart. A column was added during the prioritization 
period to identify the primary interface scales, as this would assist with coordinating with the results from the other breakout sessions. The session chair reorganized and consolidated the PIRT based on the 'Interface' column, which resulted in the 'Blade Chord' scale phenomena being separated from the 'Turbine Scale' phenomena.

The importance of blade boundary layer transition and separation was brought up several times, with separation being identified as the higher priority phenomenon. These phenomena are of the blade scale, but are very important to accurately predicting the blade load distribution, which determines the near wake development. The near wake development was identified as being important to be able to accurately predict the far-wake physics, which are important for predicting wake meandering, multiple wake merging, and turbine-turbine interaction effects.

\subsubsection{Blade Chord Scale Phenomena}

Rotor thrust as an aggregate quantity, e.g., $\mathrm{C}_{\mathrm{T}}$, was considered to be of high importance as a large-scale physical quantity, because this can be readily measured in wind tunnel experiments and field testing, and because modelers would need to aggregate more detailed predicted quantities to show agreement with this measurement. As the quantity corresponding to the next level of detail below rotor thrust, blade load distribution also was cited as being of high importance. This is important to blade force and moment distribution, and important to vorticity shedding from the blade, which produces the wake. Blade load distribution is further considered in more detail below, in some of the major PIRT items, e.g., rotational augmentation, dynamic stall, etc.

Understanding the spatial and temporal characteristics of the coherent-structure-dominated turbulent flow that arises after the approach boundary layer has interacted with the turbine blades was deemed to be of high importance. This is because the so-emerging turbulent flow provides the inflow conditions at the interface between the chord/turbine scales and the wind plant and can, thus, play an important role in determining the spatial and temporal characteristics of far wake instability. Collectively these phenomena were referred to as "blade generated energetic turbulence scales." These scales result from the intricate and inter-connected web of coherent vortices shed from the blade surfaces and the dynamically rich interactions of these vortices among themselves and the atmospheric boundary layer. Turbine generated coherent vortices include: the blade tip vortices; the vortex sheets from the blade trailing edge; the root vortices; the strong counter-rotating vortex that forms at the junction of the rotor hub with the nacelle, referred to as the nacelle vortex. Tower vortices, shed from the turbine tower, also contribute to the state of turbulence downwind of the rotor but their effect was deemed to be more significant for acoustics phenomena.

The term consolidated vorticity was used to describe turbine generated coherent vortices while the term unconsolidated vorticity was used to refer to the less understood turbulent flow that arises as the result of vortex-vortex and vortex-atmosphere interactions (blade-generated energetic turbulence scales).

The tip vortices were considered to be of high importance, due to their strongly consolidated, energetic, and persistent (at least for sufficiently low incoming turbulence intensities) nature. These qualities make the tip vortex an influential constituent of the wake, as well as more readily visualized and measured. The relative importance of the root and nacelle vortices was actively debated. One point of view was that such vortices were of medium importance, as they are less energetic and less persistent than the tip vortex. It was also pointed out, however, that the underlying physics of these vortices is still poorly understood (especially at utility scale) and, thus, we may not be able to readily conclude that they are less influential in the development of the wake. It was, in fact, emphasized that there is a rapidly expanding body of recent literature, 
based on high-fidelity numerical simulations and 3D stability analysis, pointing to a potentially significant role of the nacelle vortex on far wake development. The mechanism is related to large-scale vortex breakdown phenomena governed by the strength of the nacelle vortex, which is directly proportional to the strength of the tip vortices, and the nacelle-induced adverse pressure gradients immediately downwind of the nacelle. It was also pointed out that such turbine-scale instability may have implications at the wind plant scale as these recent studies further suggest that the energetic nacelle vortex may interact with the rotor tip shear layer in ways that can augment the intensity of far wake meandering. It was underscored, however, that the relative importance of these phenomena as a function of turbine geometric characteristics and inflow turbulence structure and intensity is not presently well understood. It was agreed that all rotor coherent vortices collectively contribute to the blade generated energetic turbulence scales, but the details of these interactions are not well understood at this point.

The unconsolidated nature of blade generated energetic turbulence scales led to some uncertainties about suitable measurement techniques. However, dense spatial distributions of elevated bandwidth 3-D probe measurements acquired in statistically significant fashion were considered useful. In addition, extended-field techniques like PIV were also deemed very significant since they can uncover and quantify coherent dynamics in the turbine wake and elucidate the underlying instabilities that govern the transition from consolidated vortices to the more complex state of unconsolidated vorticity. It was noted, however, that there are considerations of data density and frame size relative to HFM validation needs that need to be taken into account. Computational modelers identified specific methods and challenges for resolving, preserving, and identifying these vortices in simulated flowfields in various use cases and for validation exercises.

Unsteady inflow effect referred to the inflow turbulence naturally present in atmospheric inflows, and was considered of high importance because of direct influences on the blade aerodynamics and on the wake. These influences will need to be included in HFM modeling and will need to be replicated in some wind tunnel experiments. It is important to note that another prominent feature of atmospheric inflows, vertical/horizontal shear distributions, did not merit mention or discussion in this breakout session. With respect to unsteady inflow effect, scales of gust/turbulence were specifically mentioned as key supporting considerations. Comments touched on the need to unify turbulence intensity and scales between wind tunnel and SWiFT testing, to achieve realistic and useful conditions for HFM validation. Also mentioned was the ability/need for wind tunnel experiments to include the baseline zero/low turbulence or "laminar inflow" condition. It was also noted, however, that the spatial persistence and instability mechanisms of blade-generated coherent vortices can be greatly influenced by the intensity of atmospheric turbulence and atmospheric stability. Thus, studies of such phenomena at laboratory scale need to be carried out under conditions that replicate as closely as possible real-life atmospheric conditions.

Boundary layer here refers to the turbine blade boundary layer, instead of the atmospheric boundary layer. The blade boundary layer generally was considered to be of high importance, because it acts as the source or foundation for blade flow field development. Since turbine blade operation generally produces inherently unsteady flow fields, unsteady boundary layer physics was specified over and above routine steady boundary layer interactions. Due to the strong influence on flow field development and on HFM formulations and validation, it was considered important to experimentally measure blade boundary layer state, to include laminar/turbulent and attached/separated. Although not considered generally important for blade load production, tighter focus on the blade leading and trailing edge regions was considered useful for blade aeroacoustics. 
Rotational augmentation was specifically and individually called out as having high importance to the PIRT for two reasons. First, the rotationally augmented flow field is known to occur routinely during low yaw error operation on MW class turbine blades, when separation effects occur in the rotating frame under centrifugal and Coriolis influences. Second, occurrence of rotational augmentation significantly amplifies blade force and moment production relative to more simplistic though less realistic blade flow fields. Rotational augmentation was considered to be challenging for measurement and for computation, because it generally is significantly three-dimensional and possesses a substantial time varying character.

Dynamic stall also was explicitly identified as being highly important in the PIRT for the same two reasons as rotational augmentation. First, the dynamically stalled flow fields occur frequently during even modest yaw error conditions and persist throughout the elevated yaw error range on MW class turbines. Second, dynamic stall occurrence transiently, though substantially, amplifies blade force and moment production relative to more innocuous steady flows. Dynamic stall was considered to be demanding in terms of both measurement and computation, because it is inherently highly unsteady, generally is strongly three-dimensional, and very nonlinear because of pronounced viscous effects. In addition to dynamic stall, unsteady flows driven by turbulent inflows or turbine operating conditions can occur at angles of attack below stall, but nonetheless produce challenging flow fields.

Aeroelasticity was considered to be highly important and worth including in the PIRT because of alterations to blade aerodynamics in the presence of and coupled with structural dynamics. Along these lines, aerostructural stability relevant to very long, flexible future blades was identified. Aerodynamic considerations specific to aeroelasticity that were identified in the breakout included blade-to-blade aerodynamic influences and individual blade aerodynamic response consistent with the work of Theodorsen. It should be noted that structural dynamics in and of itself, being nonlinear elastodynamics, was not considered to be a challenging area for measurement or for computation in A2e. Likewise, material nonlinearities and failure were not considered in breakout session discussions, because these were not identified as relevant A2e topics.

Blade flow control was considered to be of only medium importance by the breakout, notwithstanding potential augmentations to turbine performance, reduction of acoustic signature, and attenuation of adverse loads. Moreover, the confusing breadth of flow control methodologies coupled with the general lack of demonstration experience with these technologies in wind energy, led the breakout panel to consider blade flow control to be of medium importance and thus not to be substantially considered in A2e.

Aeroacoustics seemed to be difficult to place within the A2e framework, and importance was not ranked in the high-to-medium range. Breakout session panelists acknowledged the importance of aeroacoustics to wind turbine deployment and operation. However, computational aeroacoustics experts in the breakout said that blade aeroacoustics physics and computational methodologies were relatively well established, and agreed that targeted validation data could be of use. Though there was relatively limited discussion about the specific measurements needed to support computational aeroacoustics, brief comments were made that blade flow field characterizations concentrated near the leading and trailing edges would be most useful.

Icing was acknowledged as a significant influence on blade aerodynamics and structural dynamics. However, in consideration of the projected marginal contributions to A2e and the significant added difficulties associated with measurement and computation, icing was considered to be of low importance on the PIRT. 


\subsubsection{Turbine Scale $\Leftrightarrow$ Blade Chord Scale Phenomena}

This session addressed the interactions and integrations among phenomena at the turbine scale and at the blade/chord scales that are important for wind turbine and wind plant function.

The session began with an overview by the session lead of issues and scales that represent this interface. The "Phenomena Identification \& Ranking Table" (PIRT) was partially prepopulated and organized in broader categories of relevant phenomena with specific relevant categories organized under each. We reviewed the initial categorizations and discussed modifications, deletions and additions. The attendees decided that, after modification/reduction/addition, that assessment of model adequacy (physics, code, validation) should be made at the level of the broader phenomenological groupings and that the more specific phenomena within each grouping would be specifically identified under each group. The finalized categories are listed below with a summary of discussion under each topic. Much discussion took place as we moved systematically through each of the grouped phenomena and ranked for each grouping (1) the importance of the phenomena for applications; and then the current levels of knowledge/advancements in (2) understanding the physical processes related to the phenomena and their modeling, (3) the extent of existing algorithm and code capability to predict the phenomenon, and (4) the existence of data to validate model development that address the phenomena..

Integrated Blade Loads. Given that wind turbine power derives from sectional loads integrated over the blades and the integration among the three blades at the hub, the phenomena under this topic are regarded as (very) high importance and should include both time and spatially-varying loadings. The phenomena include local blade boundary layer phenomena, including separation and dynamic stall, rotational augmentation, tip flow and associated losses, and hub-blade interactions. Whereas the group felt that specific characterization and modeling of the drivetrain is not within the scope of the HFM A2e effort, lower order models may be required for analysis of phenomena related to integrated loads across the three blades at the hub in context with power generation, deleterious transient loadings, and controls (below). There was also recognition of the importance of near-wake formation, including vortex formation and breakdown. The physics associated with the phenomena that underlie integrated blade loads were regarded as partially understood, but serious gaps exist, while the current ability to predict important details with HFM and availability of validation data were regarded as far from where we need to be.

Blade and Tower Deformations. There was discussion surrounding the question whether blade deformation should be regarded as a secondary or primary effect relevant to applications. In the end, the elastic responses of the blades to space-time variable winds were regarded as primary and the phenomena were regarded with high importance both for wind turbine function and wind plant function. Whereas understanding of the physics and modeling of aeroelastic deformations was regarded as at a high level by the group, validation data specific to wind turbine applications are lacking.

Tower deformations, in contrast, were regarded as significant but not at the same level of importance as blade deformations to applications. Furthermore the physics, prediction and validation capabilities were regarded as high.

Fluid-Structure Interactions. We decided to distinguish between the physics and modeling of the interaction between the nonsteady flow and elastic response, and the knowledge and modeling of elastic structures. The group regarded the physics and importance of the interactions between flow-generated loads and elastic response of blades as very important, with serious gaps in knowledge of important physical phenomena and low current capability to predict, and therefore in need of major advancements.

Atmospheric Turbulence Inflow and Wake Inflow from Upstream Wind Turbines 
The attendees agreed with the high importance of both understanding and predicting the wide ranges of spatially the temporally varying inflow velocity fields. These we separated into two overall topics that integrate: characterization of atmospheric microscale turbulence inflow, and characterization of downstream wake development of upstream wind turbines impacting downstream wind turbines and the interaction between atmospheric motions and wakes in regards wake meandering and control. With respect to wake formation, we have agreed that there should be a distinction between near-wake formation (below) and far wake development as relevant to inflow to downstream wind turbines. These are obviously related issues, but whereas far-wake is particularly relevant to wind turbine response within wind plants, the near-wake is important both to the development of the far-field and to the prediction of blade response on individual wind turbines. Understanding of the atmospheric turbulence contributions to the inflow are regarded as partially understood with the ability to predict canonical equilibrium ABL states using LES, but much is not understood concerning the impacts of mesoscale weather on microscale turbulence and its impact on wind turbine response, and validation data are lacking. Whereas the importance of wake inflows is high, both physical understanding and modeling capabilities are lacking, as are validation data.

Near-wake Formation and Flow. Detailed understanding and prediction of near-wake formation is important for accurate prediction of sectional and integrated blade loadings, and may be important for details of far wake formation as inflow to downstream wind turbines. Current physical understanding is regarded as high, but the challenges to prediction are high, especially in regards to dynamic resolution requirements, so although predictive capabilities exist, technical advances are required. Validation data are seriously lacking.

Tower-Rotor-Nacelle Interactions. It was pointed out the lack of understanding, and ability to predict, important issues surrounding the interactions between the tower, rotor and nacelle centers on the complexity of integrating many phenomenon with complex geometry effects. Whereas there exists some level of understanding of the important dynamical interactions, there is also a great lack of knowledge and consequent need for improved predictive capabilities and the development of data specific to validate predictions of complex interactions between the tower, the rotor and the nacelle in the loading response of wind turbines within wind plans.

Aeroacoustics on Rotating Turbine Blades. The argument was made that aeroacoustics as a field is in an advanced state, but that rotating nature of the sources in noise generation and propagation from wind turbines create special issues in physics that, depending on the specific questions being asked, can have significant gaps. Whereas methods to predict noise from rotating sources have been developed within the rotorcraft community, applications to wind turbine and wind plants contain many challenges and gaps. Thus, code development and validation data were regarded as important needs.

Controls-related Phenomena. The group felt strongly that the many various phenomena that must be considered in the application of wind turbine and wind plant controls, particular the control of pitch and yaw to both increase power capture and reduce component failures, are of high importance, but that this is an interdisciplinary topic that integrates both at the turbine-bladechord level and at the discipline level. There is perhaps a special need to address prediction of the phenomena and the generation of validation data integrated with the wind turbine/park controls community. This group assessed the knowledge of the physics as partial and with important gaps, and assessed the predictive capabilities that combine HFM of blade and turbine loadings with wind turbine control methods as low. It was pointed out that proprietary issues with OEMs are one of the challenges to address with controls-related issues. 


\subsubsection{Validation Data Requirements}

Using PIRTs collapsed from the Turbine Scaleand the Blade Chord Scale as well as their interface, data requirement needs were identified. In addition, the type of experiment and specialized instrumentation needs were discussed. In this section, the measurements needed for the different data required are presented by category of experiment.

The phenomena identified in the PIRT for which validation data are required were broken down into more general categories for the purpose of determining what validation data are required.

The six categories include the following:

- Blade Aero / Wake Generation/ Propagation,

- Boundary Layer Physics,

- Blade Flow Phenomena,

- Unsteady Inflow Effects,

- Tower/Nacelle/Rotor Wake interactions and Acoustics, and

- Aeroelasticity.

As might be expected there was significant overlap in the validation measurement needs for each of these areas. Table 2 provides a high-level summary of the measurement needs (e.g. velocity measurements, surface measurements, etc.) are provided in. In addition, special requirements (e.g. number of velocity components needed, spatial and temporal resolution requirements, etc,), complexity (2-D, 3-D, rotating), and test types (e.g. wind tunnel/field) were identified. Note that not all tests in a phenomena category will require all the measurements identified in the table, but the table can be thought to identify the range of needs for that particular category. In more detailed experiment planning, details of the experiment and which phenomena they address will dictate the instrumentation needs for that particular experiment.

Table 2. Summary of measurement needs for the turbine/blade scale

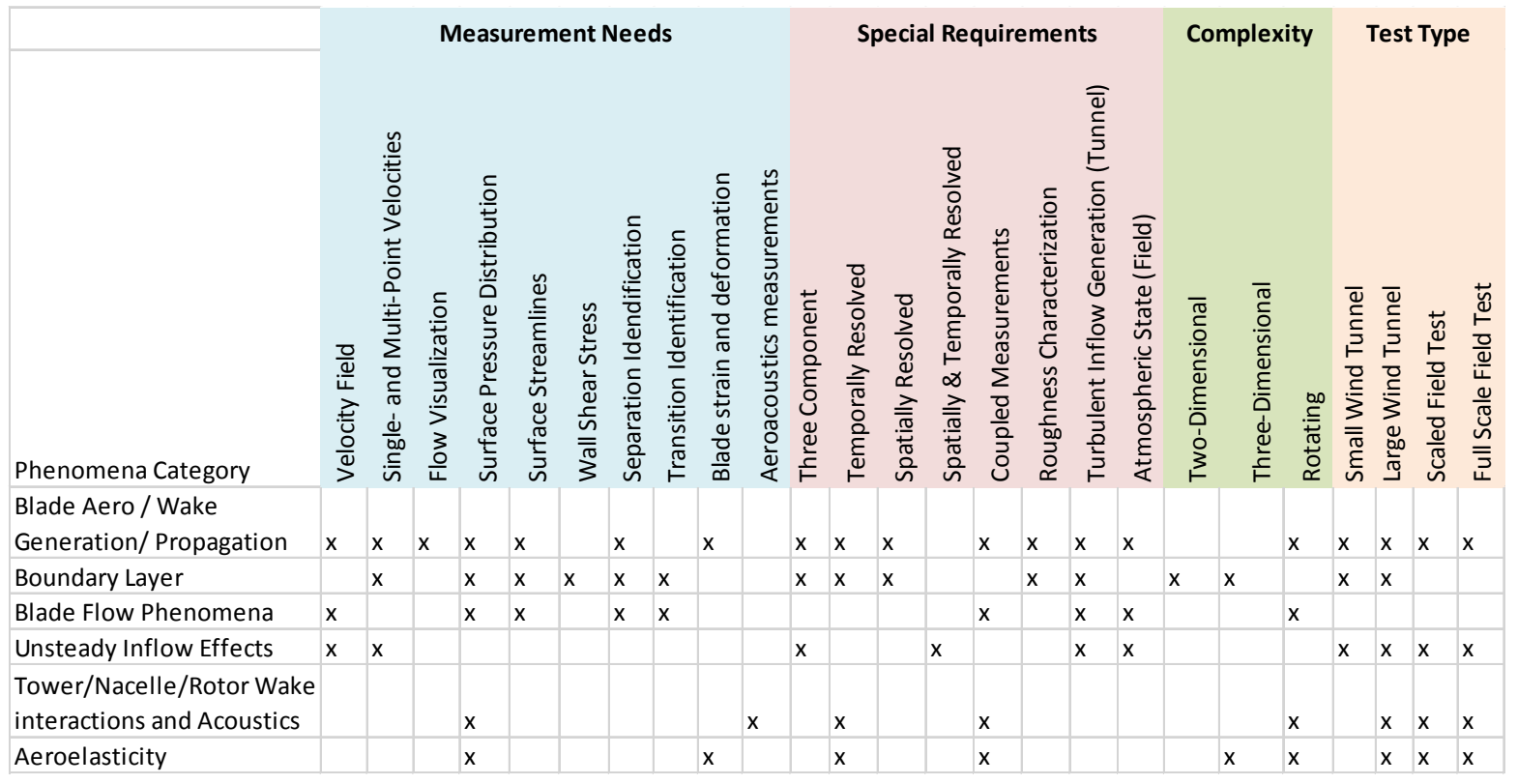

In our discussion of the measurement needs, some common themes were identified that include the following. 
- Measurement of inflow conditions suitable for use of measurements for validating computations is necessary for all tests.

- Data from tests at various levels of complexity (existing, planned, or future tests) are likely needed to provide a full validation data suite.

- Non-intrusive measurements should be performed in those flows with features (e.g. vortices) that are sensitive to probes.

- Scaling effects should be considered where possible. For example, multiple tests of similar phenomena at different Reynolds numbers should be undertaken.

- For field tests at industrial scale, leveraging SCADA systems should be undertaken, and other missing measurements should be added as needed.

In addition to identifying needs and potential tests, instrumentation requirements outside those considered "routine" were also identified:

- Time-resolved pressure measurements: both local (taps) and global (e.g Pressure sensitive paint),

- Blade shape/property characterization including time-dependent position,

- Flow-field measurements in field tests (e.g. scanning Lidar, Doppler Global Velocimetry (DGV)) and large wind tunnels (Particle Image Velocimetry (PIV)),

- Consideration of surface measurement locations and measurement techniques necessary to capture phenomena such as transition and stall locations, and

- Aeroacoutic array for identification of noise sources in wind tunnel and field test.

Finally, data processing and analysis sufficient to provide useful validation data were considered important. Such activities could range from typical data reduction (e.g. phase averaging of pressure measurements) to more complex approaches (e.g. identifying typical turbulent structure for comparing LES with measured turbulence). 


\section{Summary}

Atmosphere to electrons (A2e) is a multi-year U.S. Department of Energy (DOE) research initiative targeting significant reductions in the cost of wind energy through an improved understanding of the complex physics governing wind flow into and through whole wind farms. Better insight into the flow physics of large multi-turbine arrays will address the plant-level energy losses, is likely to reduce annual operational costs by hundreds of millions of dollars, and will improve project financing terms to more closely resemble traditional capital projects.

High-fidelity modeling (HFM), particularly of wind-plant physics, is a key component of the A2e effort. Two aspects of HFM are the physics-based modeling and the creation of a community infrastructure to improve collaborations and the availability of code, as well as to ensure the efficient use of high-performance computing platforms. One planning meeting was convened for each of these two areas.

The first planning meeting focused on requirements and priorities for developing a predictive wind plant modeling and simulation environment. Participants assessed the state of the art in open source community codes, large-scale numerical modeling, and developed requirements and specifications for a high-fidelity modeling and simulation environment. The second planning meeting focused on wind plant physics and modeling. Participants identified prioritized quantities of interest at various scales, the experimental data necessary to validate these quantities and re-addressed software needs from a wind energy modeling perspective.

Detailed requirements and recommendations have been detailed in this report. The broader results of these workshops can be summarized as follows:

- Wind-plant scale modeling and simulation will require the full power of exascale computing and has the potential to improve significantly the understanding wind-plant physics. This in turn, is expected to have a significant impact on the wind-energy industry.

- Major gaps in wind-plant modeling include dealing with wake generation and behavior, complex terrain and surface conditions, and coupling across scales.

- Verification and validation (V\&V) must be "designed-in" to wind-plant modeling, and includes the design of experiment and experimental diagnostics.

- The linkage between V\&V, experiment and testing, data storage and archiving, and windplant design and controls will generate additional requirements and will need to be considered in more depth. Reduced-order modeling will be an important part of this interface.

- High-performance computing architectures are undergoing a significant change over the next five years. Building a community infrastructure will enable more effective use of these machines and allow the wind-energy community to leverage advances from other application community.

- The full "workflow" must be considered in the modeling and simulation environment, including, among other things, problem setup, analytics, uncertainty quantification, verification and validation and data storage and transfer.

Overall the participants did not identify any major gaps that could not be resolved and would therefor prevent the development and use of a community-based modeling and simulation capability for wind-plant scale simulation. However, it was also recognized that there must be a significant and sustained investment in this effort to effectively use leading edge highperformance computing for wind-plant modeling and simulation.

Addressing the computational research needs identified in this report will reap tremendous benefits in terms of advancing the basic science underlying alternative and renewable energy 
technologies. This will lay the groundwork for, and greatly accelerate, the scientific and technological advances that will play a major role in meeting global energy needs-sustainably and cost effectively. 


\section{Appendix: Related Documents \& Reports}

[1] "Wind Vision: A New Era for Wind Power in the United States," DOE/GO-102015-4557, March 2015.

[2] 20\% Wind Energy by 2030, Increasing Wind Energy's Contribution to U.S. Electricity Supply. (2008 July). DOE/GO-102008-2567 . www1.eere.energy.gov/windandhydro/pdfs/41869.pdf.

[3] American Wind Energy Association. (2009). Windpower Outlook 2009. From www.awea.org/pubs/documents/outlook_2009.pdf

[4] "Complex Flow," Workshop Report, January 17-18, 2012, University of Colorado, Boulder. DOE/GO-102012-3653 • MAY 2012.

[5] Hammond, S., G. Bothun, and S. Piccataggio, "Computational Research Needs for Alternative and Renewable Energy," U.S. Department of Energy Workshop Report, Rockville, MD, September 2007. (http://science.energy.gov/ /media/ascr/pdf/ programdocuments/docs/Crnare_workshop_report.pdf)

[6] U.S. Department of Energy Workshop Report: Research Needs for Wind Resource Characterization, Jointly Sponsored by: DOE Office of Science, Office of Biological and Environmental Research and DOE Office of Energy Efficiency and Renewable Energy, Wind \& Hydropower Technologies Program, Co-Chairs: S. Schreck, J. Lundquist, and W. Shaw, January 14-16, 2008, Broomfield, CO, NREL Technical Report NREL/TP-500-43521, June 2008.

[7] National Research Council. (2009). Electricity from Renewable Resources: Status, Prospects, and Impediments America's Energy Future Panel on Electricity from Renewable Resources. . http://www.nap.edu/catalog/12619.html.

[8] Simon, H., Zacharia, T., \& Stevens, R. (2010). Modeling and Simulation at the Exascale for Energy and Environment. (D. R. Meetings, Ed.) 


\section{Appendix: ModSim Environment Strategic Planning Meeting Agenda}

27-28 January 2015, Crowne Plaza Denver

Day One, Tuesday, January 27, 2015

\begin{tabular}{|c|c|c|}
\hline 7:15 AM - 8:00 AM & Check in and Continental Breakfast & \\
\hline 8:00 AM - 8:15 AM & Welcome and Meeting Goals & $\begin{array}{l}\text { Michael Sprague, } \\
\text { Program Chair }\end{array}$ \\
\hline $8: 15$ AM - 8:45 AM & A2e Overview and Wind Energy Challenges & Mike Robinson \\
\hline 8:45 AM - 9:15 AM & $\begin{array}{l}\text { Plenary Talk and Discussion: The role of high } \\
\text { fidelity modeling in A2e }\end{array}$ & Shreyas Ananthan \\
\hline 9:15 AM - 9:45 AM & $\begin{array}{l}\text { Plenary Talk and Discussion: Trends in HPC } \\
\text { environments }\end{array}$ & Paul Messina \\
\hline 9:45 AM - 10:00 AM & Break & \\
\hline 10:00 AM - 12:00 PM & $\begin{array}{l}\text { Parallel Working Sessions } \\
\text { - Breakout 1: Analysis Software Components } \\
\text { (Office) } \\
\text { - Breakout 2: Implementation of Flow } \\
\text { Physics and Numerical Discretization of } \\
\text { PDEs (Elevation) } \\
\text { - Breakout 3: Addressing Software } \\
\text { Engineering, Performance, and Scalability } \\
\text { Issues on the Road To Exascale (Park) } \\
\text { - Breakout 4: Infrastructure, Integration, and } \\
\text { Software Development Processes (Altitude) }\end{array}$ & Breakout Leads \\
\hline 12:00 AM - 1:00 PM & Working Lunch & \\
\hline 1:00 PM - 1:30 PM & $\begin{array}{l}\text { Plenary Talk and Discussion: Wind farm } \\
\text { simulations using geometry-conforming models } \\
\text { for individual wind turbines }\end{array}$ & $\begin{array}{l}\text { Jayanarayanan } \\
\text { Sitaraman }\end{array}$ \\
\hline $1: 30 \mathrm{PM}-2: 00 \mathrm{PM}$ & $\begin{array}{l}\text { Plenary Talk and Discussion: The treatment of } \\
\text { turbulence and boundaries in wind farm } \\
\text { simulations }\end{array}$ & $\begin{array}{l}\text { Philippe Spalart \& } \\
\text { Matt Churchfield }\end{array}$ \\
\hline 2:00 PM - 3:30 PM & Parallel Working Sessions: Continued & \\
\hline 3:30 PM - 3:50 PM & Break & \\
\hline 3:50 PM - 4:50 PM & $\begin{array}{l}\text { Parallel Session Breakout Reports: } 15 \text { Minutes } \\
\text { per Report }\end{array}$ & Breakout Leads \\
\hline 4:50 PM - 5:00 PM & Day 1 Closing Remarks & $\begin{array}{l}\text { Michael Sprague, } \\
\text { Program Chair }\end{array}$ \\
\hline 5:00 PM & Adjourn & \\
\hline
\end{tabular}


Day Two, Wednesday, January 28, 2015

\begin{tabular}{|c|c|c|}
\hline 7:15 AM - 8:00 AM & Continental Breakfast & \\
\hline 8:00 AM - 8:15 AM & Recap Day 1 and Goals for Day 2 & $\begin{array}{l}\text { Michael Sprague, } \\
\text { Program Chair }\end{array}$ \\
\hline 8:15 AM - 8:45 AM & $\begin{array}{l}\text { Plenary Talk and Discussion: Creating a } \\
\text { flexible, extensible and maintainable } \\
\text { community earth system model }\end{array}$ & Mariana Vertenstein \\
\hline 8:45 AM - 9:15 AM & $\begin{array}{l}\text { Plenary Talk and Discussion: Multi-Lab } \\
\text { collaborative development of ACME }\end{array}$ & Mark Taylor \\
\hline 9:15 AM - 9:45 AM & $\begin{array}{l}\text { Plenary Talk and Discussion: Multi- } \\
\text { Institutional Development of Scientific } \\
\text { Software - Lessons Learned in CASL }\end{array}$ & John Turner \\
\hline 9:45 AM - 10:00 AM & Break & \\
\hline 10:00 AM - 12:00 PM & $\begin{array}{l}\text { Parallel Working Sessions } \\
\text { - Breakout 1: Analysis Software Components } \\
\text { (Office) } \\
\text { - Breakout 2: Implementation of Flow } \\
\text { Physics and Numerical Discretization of } \\
\text { PDEs (Elevation) } \\
\text { - Breakout 3: Addressing Software } \\
\text { Engineering, Performance, and Scalability } \\
\text { Issues on the Road To Exascale (Park) } \\
\text { - Breakout 4: Infrastructure, Integration, and } \\
\text { Software Development Processes (Altitude) }\end{array}$ & Breakout Leads \\
\hline 12:00 PM - 1:00 PM & Working Lunch & \\
\hline 1:00 PM - 1:30 PM & $\begin{array}{l}\text { Plenary Talk and Discussion: Uncertainty and } \\
\text { Reliability in Computational Predictions }\end{array}$ & Robert Moser \\
\hline 1:30 PM - 3:30 PM & Parallel Working Sessions: Continued & \\
\hline 3:30 PM - 3:50 PM & Break & \\
\hline 3:50 PM - 5:10 PM & Breakout Reports: 20 Minutes per Report & Breakout Leads \\
\hline 5:10 PM - 5:30 PM & Closing Remarks & $\begin{array}{l}\text { David Womble and } \\
\text { Steve Hammond, A2e } \\
\text { HFM Co-Chairs }\end{array}$ \\
\hline
\end{tabular}

Day Three: Thursday, January 29, 2015

Meeting Organizers and Breakout Leads Only

\begin{tabular}{|l|l|l|}
\hline 8:00 AM - 12:00 PM & $\begin{array}{l}\text { Writing Time for Meeting Organizers and } \\
\text { Breakout Leads (Altitude) }\end{array}$ & \\
\hline 12:00 PM & Adjourn & \\
\hline
\end{tabular}




\section{Appendix: Wind Plant Physics and Modeling Planning Meeting Agenda}

24-25 February 2015, Double Tree-Crystal City, Washington, DC

\section{Day One, Tuesday, February 24, 2015}

\begin{tabular}{|c|c|c|}
\hline $\begin{array}{l}\text { 7:15 AM - 8:00 } \\
\text { AM }\end{array}$ & $\begin{array}{l}\text { Check in / Continental Breakfast, Washington } \\
\text { Foyer }\end{array}$ & \\
\hline $\begin{array}{l}\text { 8:00 AM - 8:30 } \\
\text { AM }\end{array}$ & Welcome, Meeting Goals and Opening Remarks & $\begin{array}{l}\text { Matt Barone, Program } \\
\text { Chair, Jose Zayas, DOE } \\
\text { Wind Program }\end{array}$ \\
\hline 8:30 AM -9:00 AM & $\begin{array}{l}\text { Plenary Talk and Discussion: A2e Overview and } \\
\text { Wind Energy Challenges }\end{array}$ & Mike Robinson \\
\hline $\begin{array}{l}\text { 9:00 AM - 9:30 } \\
\text { AM }\end{array}$ & $\begin{array}{l}\text { Plenary Talk and Discussion: Validation } \\
\text { Framework }\end{array}$ & Rich Hills \\
\hline $\begin{array}{l}\text { 9:30 AM - 9:45 } \\
\text { AM }\end{array}$ & Break & \\
\hline $\begin{array}{l}9: 45 \text { AM - 11:45 } \\
\text { AM }\end{array}$ & $\begin{array}{l}\text { Breakout Session \#1 } \\
\text { Phenomena Identification \& Ranking by Scale } \\
\text { - Group A: Mesoscale } \\
\text { - Group B: Plant Scale } \\
\text { - Group C: Turbine Scale } \\
\text { - Group D: Blade Chord Scale }\end{array}$ & \\
\hline $\begin{array}{l}\text { 11:45 AM - 12:35 } \\
\text { PM }\end{array}$ & Working Lunch & \\
\hline $\begin{array}{l}\text { 12:35 PM - 1:15 } \\
\text { PM }\end{array}$ & $\begin{array}{l}\text { Plenary Talk and Discussion: Challenges in } \\
\text { Modeling Scale Interfaces }\end{array}$ & $\begin{array}{l}\text { Jeff Mirocha and Jim } \\
\text { Brasseur }\end{array}$ \\
\hline 1:15 PM - 3:15 PM & $\begin{array}{l}\frac{\text { Breakout Session } \# \mathbf{2}}{\text { Phenomena Identification \& Ranking by Scale }} \\
\text { Interface } \\
\text { - Group A: Mesoscale }<->\text { Plant Scale } \\
\text { - Group B: Plant Scale }<->\text { Turbine Scale } \\
\text { - Group C: Turbine Scale }<->\text { Blade Chord } \\
\text { Scale }\end{array}$ & Breakout Leads \\
\hline 3:15 PM - 3:30 PM & Break & \\
\hline 3:30 PM - 5:00 PM & Breakout Session Reports & Breakout Leads \\
\hline 5:00 PM - 5:15 PM & Closing Remarks and Adjourn & Program Chair \\
\hline
\end{tabular}




\section{Day Two, Wednesday, February 25, 2015}

\begin{tabular}{|c|c|c|}
\hline $\begin{array}{l}\text { 7:15 AM - 8:00 } \\
\text { AM }\end{array}$ & $\begin{array}{l}\text { Check in / Continental Breakfast, Washington } \\
\text { Foyer }\end{array}$ & \\
\hline $\begin{array}{l}\text { 8:00 AM - 8:15 } \\
\text { AM }\end{array}$ & Recap Day 1 and Goals for Day 2 & $\begin{array}{l}\text { Matt Barone, Program } \\
\text { Chair }\end{array}$ \\
\hline $\begin{array}{l}8: 15 \text { AM }-8: 45 \\
\text { AM }\end{array}$ & $\begin{array}{l}\text { Plenary Talk and Discussion: High Fidelity } \\
\text { Modeling Efforts at Vestas: Current Work, Future } \\
\text { Projections, and Needs. }\end{array}$ & Greg Oxley \\
\hline $\begin{array}{l}8: 45 \text { AM - 9:15 } \\
\text { AM }\end{array}$ & $\begin{array}{l}\text { Plenary Talk and Discussion: High Fidelity } \\
\text { Modeling at AWS Truepower }\end{array}$ & Philippe Beaucage \\
\hline $\begin{array}{l}\text { 9:15 AM - 9:45 } \\
\text { AM }\end{array}$ & $\begin{array}{l}\text { Plenary Talk and Discussion: Current DOE Wind } \\
\text { Program Validation Experiment Plans }\end{array}$ & David Maniaci \\
\hline $\begin{array}{l}9: 45 \text { AM - 10:00 } \\
\text { AM }\end{array}$ & Break & \\
\hline $\begin{array}{l}\text { 10:00 AM - 12:00 } \\
\text { PM }\end{array}$ & $\begin{array}{l}\text { Breakout Session \#3 } \\
\text { Validation Data Requirements } \\
\text { - Group A: Mesoscale }<->\text { Plant Scale } \\
\text { - Group B: Plant Scale }<\text { - }>\text { Turbine Scale } \\
\text { - Group C: Turbine Scale }<\text { - }>\text { Blade Chord } \\
\quad \text { Scale }\end{array}$ & Breakout Leads \\
\hline $\begin{array}{l}\text { 12:00 PM - 1:00 } \\
\text { PM }\end{array}$ & Working Lunch & \\
\hline $\begin{array}{l}\text { 1:00 PM - 1:30 } \\
\text { PM }\end{array}$ & $\begin{array}{l}\text { Plenary Talk and Discussion: Outcomes from the } \\
\text { ModSim Environments Planning Meeting }\end{array}$ & Michael Sprague \\
\hline $\begin{array}{l}1: 30 \mathrm{PM}-3: 30 \\
\mathrm{PM}\end{array}$ & $\begin{array}{l}\text { Breakout Session \#4 } \\
\text { Software Requirements from the Modelers' } \\
\text { Perspective } \\
\text { - Group A: Mesoscale }<->\text { Plant Scale } \\
\text { - Group B: Plant Scale }<->\text { Turbine Scale } \\
\text { - Group C: Turbine Scale }<->\text { Blade Chord } \\
\text { Scale }\end{array}$ & Breakout Leads \\
\hline $\begin{array}{l}3: 30 \text { PM - 3:45 } \\
\text { PM }\end{array}$ & Break & \\
\hline $\begin{array}{l}\text { 3:45 PM - 5:15 } \\
\text { PM }\end{array}$ & Breakout Session Reports & Breakout Leads \\
\hline $\begin{array}{l}\text { 5:15 PM - 5:30 } \\
\text { PM }\end{array}$ & Closing Remarks and Adjourn & Program Chair \\
\hline
\end{tabular}


Day Three, Thursday, February 26, 2015

\begin{tabular}{|l|l|l|}
\hline $\begin{array}{l}\text { 8:00 AM - 12:00 } \\
\text { PM }\end{array}$ & $\begin{array}{l}\text { Writing Time for Meeting Organizers and } \\
\text { Breakout Leads } \\
\text { Location: Van Buren Room }\end{array}$ & \\
\hline 12:00 PM & Adjourn \\
\hline
\end{tabular}




\section{Appendix: January Planning Meeting Registered Attendees}

Analysis Software Components Breakout Participants:

\begin{tabular}{|l|l|l|}
\hline Last & First & Affiliation \\
\hline Barone & Matthew & Sandia National Laboratories \\
\hline Constantine & Paul & Colorado School of Mines \\
\hline Eldred & Michael & Sandia National Laboratory \\
\hline Gopalarathnam & Ashok & North Carolina State University \\
\hline Gulstad & Line & Vestas Wind Systems A/S \\
\hline Hamlington & Peter & University of Colorado, Boulder \\
\hline Jakeman & John & Sandia National Laboratories \\
\hline Kotamarthi & Rao & Argonne National Laboratory \\
\hline Lundquist & Julie & U. of Colorado \& National Renewable Energy Lab. \\
\hline Martin & Ezequiel & University of Iowa \\
\hline Mirocha & Jeff & Lawrence Livermore National Laboratory \\
\hline Moser & Robert & University of Texas at Austin \\
\hline Munoz-Esparza & Domingo & Los Alamos National Laboratory \\
\hline Stewart & James & Sandia National Laboratories \\
\hline Veers & Paul & National Renewable Energy Laboratory \\
\hline von Terzi & Dominic & GE Global Research \\
\hline
\end{tabular}

Implementation of Flow Physics and Numerical Discretization of PDEs Participants:

\begin{tabular}{|l|l|l|}
\hline Last & First & Affiliation \\
\hline Ananthan & Shreyas & US Department of Energy \\
\hline Balakrishnan & Ramesh & Argonne National Laboratory \\
\hline Churchfield & Matthew & National Renewable Energy Laboratory \\
\hline Colella & Phillip & Lawrence Berkeley National Laboratory \\
\hline Domino & Stefan & Sandia National Laboratory \\
\hline Jansen & Kenneth & University of Colorado at Boulder \\
\hline Kosovic & Branko & NCAR \\
\hline Mahesh & Krishnan & University of Minnesota \\
\hline Maniaci & David & Sandia National Laboratories \\
\hline Mavriplis & Dimitri & University of Wyoming \\
\hline Meneveau & Charles & Johns Hopkins University \\
\hline Nichols & Joseph & University of Minnesota \\
\hline Patton & Edward (Ned) & NCAR \\
\hline Persson & Per & University of California - Berkeley \\
\hline Sitaraman & Jay & U. of Wyoming and U.S Army at NASA Ames \\
\hline Slattery & Stuart & Oak Ridge National Laboratory \\
\hline Smith & Thomas & Sandia National Laboratories \\
\hline Sotiropoulos & Fotis & University of Minnesota \\
\hline Spalart & Philippe & The Boeing Company \\
\hline Sprague & Michael & National Renewable Energy Laboratory \\
\hline Sullivan & Peter & NCAR \\
\hline
\end{tabular}


Addressing Software Engineering, Performance, and Scalability Issues on the Road to Exascale Participants:

\begin{tabular}{|l|l|l|}
\hline Last & First & Affiliation \\
\hline Barker & Kevin & Pacific Northwest National Laboratory \\
\hline Brasseur & James & Penn State University \\
\hline Brown & Jed & Argonne National Laboratory/U. of Colorado \\
\hline Carpenter & Ilene & National Renewable Energy Laboratory \\
\hline Chan & Cy & Lawrence Berkeley National Laboratory \\
\hline Gray & Justin & NASA Glenn \\
\hline Johnson & Nick & US Department of Energy \\
\hline Kerbyson & Darren & Pacific Northwest National Laboratory \\
\hline Koo & Eunmo & Los Alamos National Laboratory \\
\hline McCormick & Patrick & Los Alamos National Laboratoy \\
\hline Messina & Paul & Argonne National Laboratory \\
\hline Mills & Richard & Intel Corporation \\
\hline Purkayastha & Avi & National Renewable Energy Laboratory \\
\hline Streitz & Fred & Lawrence Livermore National Laboratory \\
\hline Wells & Jack & Oak Ridge National Laboratory \\
\hline
\end{tabular}

Infrastructure, Integration, and Software Development Process Participants:

\begin{tabular}{|l|l|l|}
\hline Last & First & Affiliation \\
\hline Brewster & Keith & Ohio University \\
\hline Carrica & Pablo & University of Iowa \\
\hline Dykes & Katherine & National Renewable Energy Laboratory \\
\hline Gable & Carl & Los Alamos National Laboratory \\
\hline Graf & Peter & National Renewable Energy Laboratory \\
\hline Kendall & Richard & DoD HPCMC \\
\hline McCaskey & Alex & Oak Ridge National Laboratory \\
\hline Michalakes & John & NOAA/NWS/NCEP, Environmental Modeling Center \\
\hline Moriarty & Patrick & National Renewable Energy Laboratory \\
\hline Potsdam & Mark & US Army \\
\hline Robinson & Mike & US Department of Energy \\
\hline Taylor & Mark & Sandia National Laboratories \\
\hline Turner & John & Oak Ridge National Laboratory \\
\hline Vertenstein & Mariana & NCAR \\
\hline
\end{tabular}




\section{Appendix: February Planning Meeting Registered Attendees}

Mesoscale/Plant Scale Participants:

\begin{tabular}{|l|l|l|}
\hline Last & First & Affiliation \\
\hline Ananthan & Shreyas & \\
\hline Archer & Cristina & \\
\hline Beaucage & Philippe & \\
\hline Berg & Larry & Pacific Northwest National Laboratory \\
\hline Finley & Cathy & \\
\hline Freedman & Jeff & \\
\hline Hamlington & Peter & NCAR \\
\hline Haupt & Sue & \\
\hline Lungo & Giacomo V. & \\
\hline Kotamarthi & Rao & DOE Office of Science, ASCR \\
\hline Lauzon & Carolyn & \\
\hline Martin & Ezequiel & \\
\hline Meneveau & Charles & Lawrence Livermore National Laboratory \\
\hline Mirocha & Jeff & National Renewable Energy Laboratory \\
\hline Moriarty & Patrick & \\
\hline Pryor & Sara & \\
\hline Shen & Lian & Oak Ridge National Laboratory \\
\hline Turner & John & \\
\hline Ueda & Yuko & National Renewable Energy Laboratory \\
\hline Veers & Paul & \\
\hline Venayagamoorthy & Karan & NOAA \\
\hline Wilczak & Jim & \\
\hline
\end{tabular}

\section{Plant Scale / Turbine Scale Participants:}

\begin{tabular}{|l|l|l|}
\hline Last & First & Affiliation \\
\hline Barthelmie & Rebecca & \\
\hline Bazilevs & Yuri & \\
\hline Bhaganagar & Kiran & \\
\hline Bleeg & James & \\
\hline Carrica & Pablo & \\
\hline Carrigan & Travis & \\
\hline Chamorro & Leonardo & \\
\hline Chatelain & Philippe & National Renewable Energy Laboratory \\
\hline Churchfield & Matt & National Renewable Energy Laboratory \\
\hline Dykes & Katherine & Sandia National Laboratories \\
\hline Ennis & Brandon & Los Alamos National Laboratory \\
\hline Linn & Rod & \\
\hline McKillip & Robert & \\
\hline Oxley & Gregory & \\
\hline Peet & Yulia & \\
\hline Ponta & Fernando & \\
\hline Resor & Brian & \\
\hline Schmitz & Sven & \\
\hline
\end{tabular}




\begin{tabular}{|l|l|l|}
\hline Sprague & Michael & National Renewable Energy Laboratory \\
\hline von Terzi & Dominic & GE \\
\hline Wells & Jack & Oak Ridge National Laboratory \\
\hline White & Jon & Sandia National Laboratories \\
\hline Wyman & Nick & \\
\hline
\end{tabular}

Turbine Scale / Blade Scale Participants:

\begin{tabular}{|l|l|l|}
\hline Last & First & Affiliation \\
\hline Balakrishnan & Ramesh & Argonne National Laboratory \\
\hline Blaylock & Myra & \\
\hline Brasseur & James & Penn State University \\
\hline Brentner & Ken & \\
\hline Butterfield & Sandy & \\
\hline Colella & Phillip & Lawrence Berkeley National Laboratory \\
\hline Duque & Earl & \\
\hline Duraisamy & Karthik & \\
\hline Hsu & Ming-Chen & \\
\hline Maniaci & David & Sandia National Laboratories \\
\hline Martin & Michael & DOE Office of Science, ASCR \\
\hline Modarres-Sadeghi & Yahya & \\
\hline Naughton & Jonathan & \\
\hline Nichols & Joe & \\
\hline Pannala & Sreekanth & Oak Ridge National Laboratory (now at SABIC) \\
\hline Sarkar & Sutanu & \\
\hline Schreck & Scott & National Renewable Energy Laboratory \\
\hline Slattery & Stuart & Oak Ridge National Laboratory \\
\hline Smith & Marilyn & \\
\hline Smith & Thomas & Sandia National Laboratory \\
\hline Sotiropolous & Fotis & University of Minnesota \\
\hline Streitz & Fred & \\
\hline Whitehouse & Glen & \\
\hline & & \\
\hline
\end{tabular}

